\title{
SciNOvA: A Measurement of Neutrino-Nucleus Scattering in a Narrow-Band Beam
}

\author{
J. Paley, Z. Djurcic \\ Argonne National Laboratory, Argonne, IL \\ D. Harris, R. Tesarek \\ Fermi National Accelerator Laboratory, Batavia, IL \\ G. Feldman \\ Harvard University, Cambridge, MA \\ L. Corwin, M.D. Messier, N. Mayer, J. Musser, J. Paley, R. Tayloe, J. Urheim \\ Indiana University, Bloomington, IN \\ M. Sanchez \\ Iowa State University, Ames, IA \\ K. Heller \\ University of Minnesota, Minneapolis \\ S. Mishra, X. Tian \\ University of South Carolina, Columbia, SC \\ H. Meyer \\ Wichita State University, Wichita, KS \\ P. Vahle \\ The College of William and Mary, Williamsburg, VA
}

October 15, 2010

\begin{abstract}
We propose to construct and deploy a fine-grained detector in the Fermilab NOvA 2 GeV narrow-band neutrino beam. In this beam, the detector can make unique contributions to the measurement of quasi-elastic scattering, neutral-current elastic scattering, neutral-current $\pi^{0}$ production, and enhance the NOvA measurements of electron neutrino appearance. To minimize cost and risks, the proposed detector is a copy of the SciBar detector originally built for the K2K long baseline experiment and used recently in the SciBooNE experiment.
\end{abstract}




\section{Contents}

1 Executive Summary $\quad 3$

2 SciNOvA physics $\quad 4$

2.1 Charged-Current Quasielastic Scattering . . . . . . . . . . . . . . 5

2.1.1 A search for 2-nucleon correlations in CCQE interactions . . . . . . . . . 8

2.2 Neutral current channels . . . . . . . . . . . . . . . . . . . . . . . . . 12

2.2.1 Neutral current $\pi^{0}$ production . . . . . . . . . . . . . . . . . 12

2.2.2 Neutral current elastic scattering . . . . . . . . . . . . . . . . . . . . 12

2.3 Comparison to wide-band beam measurements . . . . . . . . . . . . . 12

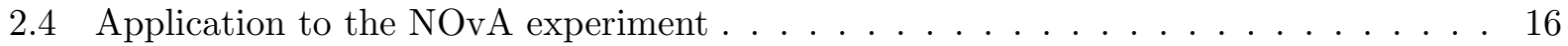

2.4.1 "Double scan" by event resampling . . . . . . . . . . . . . . . 18

2.4 .2 Numerical example . . . . . . . . . . . . . . . . . . . . . . . . 20

3 Experiment $\quad 21$

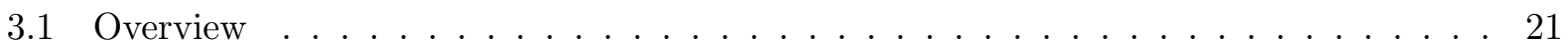

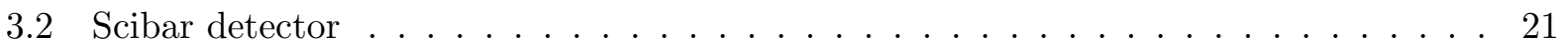

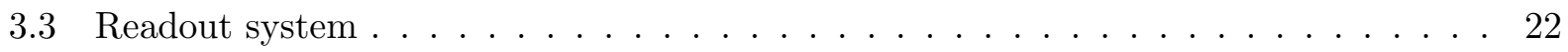

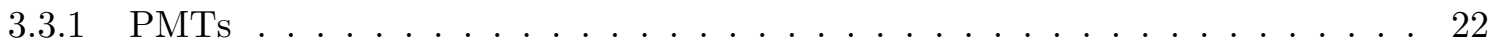

3.3 .2 IRM boards . . . . . . . . . . . . . . . . . . . . . 24

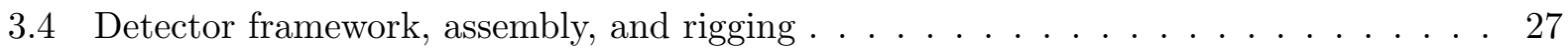

4 Cost and schedule $\quad 31$

4.1 Cost estimate . . . . . . . . . . . . . . . . . . . . 32

4.2 Schedule . . . . . . . . . . . . . . . . . . . . 34 


\section{Executive Summary}

Since the discovery of neutrino oscillations, there has been a world-wide experimental effort in particle physics to use oscillations to measure the fundamental properties of the neutrino and ultimately explore the possibility that neutrinos are responsible for the matter/anti-matter asymmetry in the universe. These experiments (K2K, MiniBooNE, MINOS, T2K, NOvA, and future experiments at a Deep Underground Science and Engineering Laboratory) require detailed knowledge of neutrinonucleus interactions to avoid being limited by uncertainties in the underlying neutrino-nucleus scattering process.

The upcoming NOvA experiment will be the cornerstone of the domestic high-energy physics program over the next decade and will search for $\nu_{\mu} \rightarrow \nu_{e}$ and $\bar{\nu}_{\mu} \rightarrow \bar{\nu}_{e}$ oscillations. For these efforts to be successful, we must improve the precision of neutrino-nucleus scattering measurements. Several experiments (Minerva, SciBooNE, e.g.) have undertaken measurement programs, but they use beams with a relatively large energy spread ("wide band") and hence have little a priori knowledge of the incident neutrino energy. NOvA, however, uses a narrow-band neutrino beam centered at $2 \mathrm{GeV}$. A fine-grained detector in this narrow-band beam would provide a unique opportunity to measure neutrino cross sections with a priori knowledge of the neutrino energy. The proposed fine-grained detector, "SciNOvA", is a copy of the SciBar detector, originally built for the K2K experiment and recently used in the SciBooNE experiment. In the region near $2 \mathrm{GeV}$, this detector would record a large sample of approximately $1 \mathrm{M}$ events per year.

The narrow energy spread of the NOvA beam gives many advantages for neutrino scattering measurements over the more common wide-band beams. First, it will allow for the suppression of backgrounds to measurements of quasielastic (QE) neutrino interactions. Recent data indicates we may not understand this process as well has had been historically assumed. Current results do not agree with the bubble chamber results from the 1970's and 80's. The source of this discrepancy is unknown; it may be due to poorly understood physics in the nucleus, nucleon, or due to experimental effects. A low-background measurement of $\mathrm{QE}$ scattering at $2 \mathrm{GeV}$ with the SciNOvA experiment would fall exactly in the region between the current measurements. Further, due to fine segmentation, the SciNOvA detector would have a low threshold for detecting recoil protons, enabling a search for di-nucleon QE final states - a "smoking gun" for one proposed solution to the discrepancies seen in QE scattering.

Additionally, the narrow band beam is ideal for measurements of neutral-current (NC) interactions. In these events, an undetected neutrino carries away some of the event energy. Specifically, SciNOvA can measure the rate and spectrum of $\pi^{0}$ production in NC interactions which is an important background for searches for $\nu_{\mu} \rightarrow \nu_{e}$ oscillations.

Finally, measurements made by SciNOvA will provide an important service for the neutrino oscillation measurements of NOvA for a relatively small investment of money and effort. Since the detector will be located in the same beam as the NOvA detector and will be composed of the same material, this higher-resolution detector will enable a robust, data-driven estimate of the instrumental backgrounds to the electron neutrino appearance and muon neutrino disappearance searches, increasing the precision of and confidence in those results.

The total cost of this proposal is $\$ 2.4 \mathrm{M}$ and covers scintillator production, photomultipliers, the readout system, and detector installation. As we plan to reproduce an existing detector, the costs are well known, the project risks are low, and the project may move expeditiously. While the proposed readout system is new, it has been extensively prototyped under a previous NSF grant. In addition to its physics program, the construction of SciNOvA is the right scale to provide excellent first-hand training of graduate and undergraduate students in the construction and operation of high energy and nuclear physics experiments. 


\section{$2 \quad$ SciNOvA physics}

The installation of a fine-grained $(1 \mathrm{~cm} \times 2 \mathrm{~cm}$ pixel size) SciBar detector in front of the NOvA near detector in the NOvA narrow-band neutrino beam will enable new insight into the physics of neutrino-nucleus interactions. This will both help the NOvA oscillation program with measurements of oscillation background processes (in particular $\mathrm{NC} \pi^{0}$ production) and will allow for an investigation of the anomalously large cross section values recently reported by MiniBooNE. The data would complement that of other experiments in this energy range and will be valuable additional information for both the underlying physics and for better understanding of neutrino oscillation background processes.

The event rates for the various charged- and neutral-current channels are shown in Table 1 and the interaction rate as a function of neutrino energy is shown in Fig.1. This energy distribution is particularly well-suited for several interesting physics channels, as discussed below.

\begin{tabular}{lll}
\hline \hline & Charged-current & Neutral-current \\
\hline elastic & 220 & 86 \\
resonant & 327 & 115 \\
DIS & 289 & 96 \\
coherent & 8 & 5 \\
\hline total & 845 & 302 \\
\hline$\nu+A \rightarrow \pi^{0}+X$ & 204 & 106 \\
\hline \hline
\end{tabular}

Table 1: Event rates for elastic, resonant, deep-inelastic (DIS) and coherent neutrino scattering processes for one year of running in neutrino mode for the 10 ton SciBar fiducial volume. Units are 1000's of events. Event rates for inclusive $\pi^{0}$ production are also shown. Rates were calculated using the GENIE [1] neutrino generator.

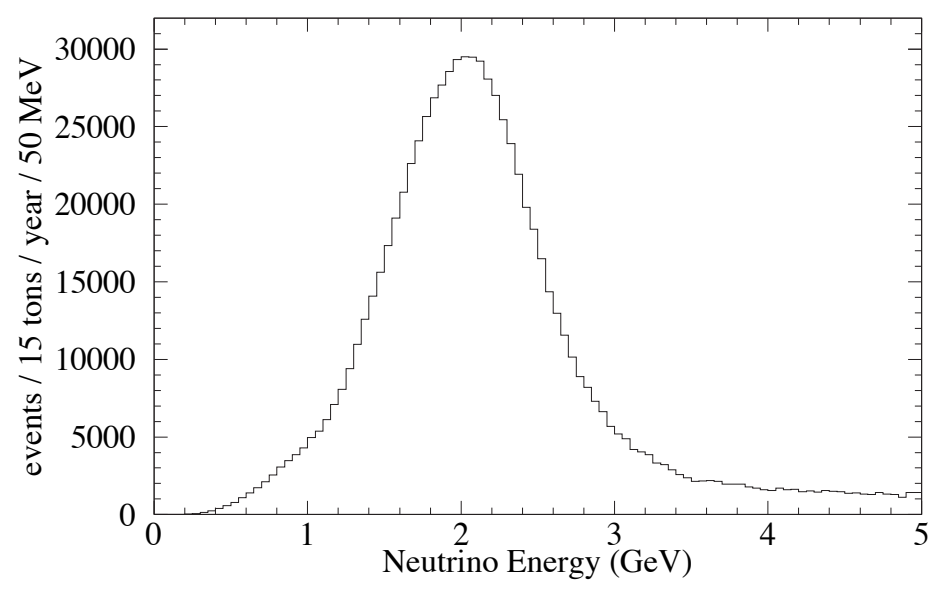

Figure 1: Event rate as a function of neutrino energy for a detector in the near hall in the NOvA narrow-band neutrino beam. 


\subsection{Charged-Current Quasielastic Scattering}

A thorough understanding of the charged-current quasielastic scattering (CCQE) process of neutrinos on (bound) nucleons $(\nu+n \rightarrow \mu / e+p$ and $\bar{\nu}+p \rightarrow \mu / e+n)$ in the $1 \mathrm{GeV}$ energy region is important for neutrino experiments as it is the cleanest detection reaction for both appearance and disappearance searches. Ultimately, we need to completely understand the physics behind this interaction in order to make precision oscillation measurements.

A puzzle has recently arisen in the charged-current quasielastic $\left(\nu_{\mu}+n \rightarrow \mu^{-}+p\right)$ results from the MiniBooNE experiment running on the FNAL Booster Neutrino Beamline (BNB). Results from previous CCQE experiments (for a recent review see Ref. [2]), performed predominantly with bubble chambers on light nuclei, yield an axial mass, $M_{A}=1.03 \pm 0.02 \mathrm{GeV}$ [3]. The axial mass parametrizes the $Q^{2}$ (4-momentum transfer) evolution of the axial form factor. The MiniBooNE data contains more events at larger $Q^{2}\left(>0.4 \mathrm{GeV}^{2}\right)$ than can be reconciled with this previously measured value of $M_{A}$ and results in a value of $M_{A}=1.35 \pm 0.20 \mathrm{GeV}$ [4].

The MiniBooNE value for $M_{A}$ quoted above is extracted in an analysis that only considers the relative shape of the $Q^{2}$ distribution as is conventional in this procedure. It may be that nuclear effects due to the binding of the target nucleons within carbon are the cause for this apparent disagreement with previous results, which utilized mainly hydrogen or deuterium targets (see, for example, Ref. [5]). However, in a recent extraction of the absolute cross section for the CCQE process, MiniBooNE has reported total cross section values as a function of neutrino energy that are large compared to those calculated using $M_{A}=1.03 \mathrm{GeV}$. The measured total cross section is consistent to within $10 \%$ (the error on overall normalization) of the expected cross section with $M_{A}=1.35 \mathrm{GeV}$. Nuclear effects can give a larger effective $M_{A}$ in the $Q^{2}$ shape of the data [6] but these effects reduce the total cross section, not enhance it. It may be that the larger measured $M_{A}$ values in both $Q^{2}$ shape and reaction rate are a coincidence, but further investigation is warranted.

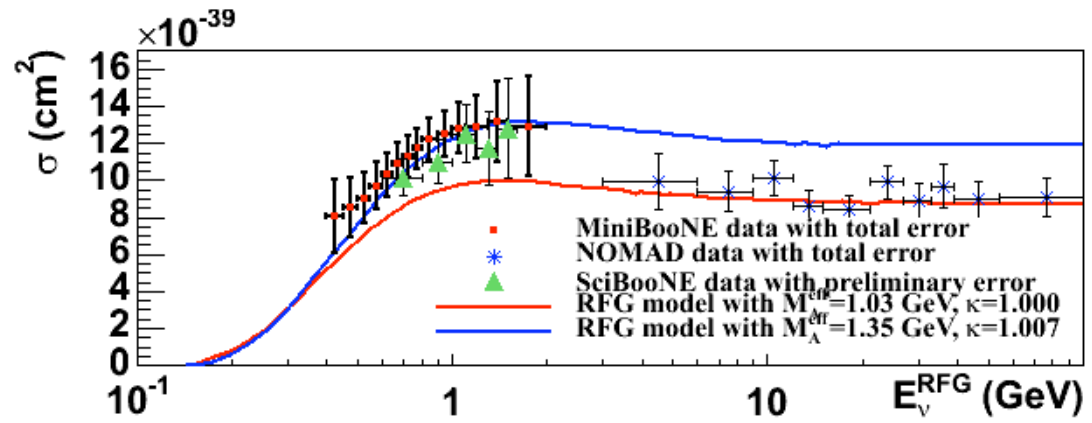

Figure 2: Measurements of quasi-elastic neutrino cross-section as a function of energy.

Recent results from other experiments have added further information to the situation, but have not clarified it. Preliminary results from SciBooNE [7], using the SciBar detector in the FNAL BNB, also show a total CCQE cross section larger than that expected from $M_{A}=1.03$. However, recent results from NOMAD [2] running at higher neutrino energies show total cross section values consistent with the previous world average $M_{A}$. The MiniBooNE, SciBooNE, and NOMAD results for the total CCQE cross section are shown together as a function of neutrino energy in Fig. 2. As is evident from that figure, it would be desirable to investigate the CCQE reaction in a beam of $E_{\nu} \approx 2 \mathrm{GeV}$, exactly that of the NOvA narrow-band beam.

We have investigated how the SciNOvA effort would add to this experimental situation. By utilizing the error analysis of the recent MiniBooNE CCQE analysis [4], we estimated the systematic 
errors that would result on a SciNOvA measurement of the CCQE total cross section. The results are summarized in Fig. 3. The systematic errors in MiniBooNE were dominated by uncertainties in the neutrino flux and the background from charged-current $\pi^{+}\left(\mathrm{CC} \pi^{+}\right)$production. For the purposes of this study we used a simple factor of $10 \%$ for the absolute flux uncertainty. In actuality, the flux error is a function of neutrino energy and grows in the tails ("off-peak") of the flux distribution, however, the $\mathrm{CC} \pi^{+}$error dominates off peak, therefore, this simplification is justified. The $\mathrm{CC} \pi^{+}$background error is due to $\mathrm{CC} \pi^{+}$events in which the $\pi^{+}$is absorbed and there is considerable uncertainty in the cross section for this process. We have used the same error employed in the MiniBooNE analysis which is taken from errors on the available pion absorption data [4]. The $\mathrm{CC} \pi^{+}$background is then broken down into two components: $\mathrm{CC} \pi^{+}$with pion absorption in the nucleus and $\mathrm{CC} \pi^{+}$with pion absorption in the detector. For the former, the NUANCE event generator [8] is used to model this process, for the latter it was estimated that $10 \%$ of pions are lost in the detector, as seen by MiniBOONE. The same $25 \%$ uncertainty on $\pi^{+}$absorption was used for both components. Statistical errors were not considered in this analysis as they will be negligible in the energy region of concern.

As can be seen in Fig. 3, the systematic error on the total cross section is dominated by the neutrino flux error in the region of the peak neutrino energy. The $\mathrm{CC} \pi^{+}$background errors dominate at energies below from the flux peak. This is because there is substantial "feed down" of $\mathrm{CC} \pi^{+}$from higher to lower reconstructed neutrino energy causing this background to become a larger fraction of the event sample at lower energy. The important result from this study is that, at the flux peak of $2 \mathrm{GeV}$, the error on the measured total cross section for the CCQE process is approximately $10 \%$ and is dominated by the flux error. This will allow an accurate measurement of the total CCQE cross section in the region just above the MiniBooNE measurements at $2 \mathrm{GeV}$. A $10 \%$ measurement of the cross section is satisfactory given the $30 \%$ discrepency of the MiniBooNE data with expectation. A second important conclusion from this analysis (See Sec. 2.3) is that the $\mathrm{CC} \pi^{+}$backgrounds grow quickly at energies below the flux peak. This will limit the accuracy of CCQE measurement from a wide-band beam at energies below the flux peak. 

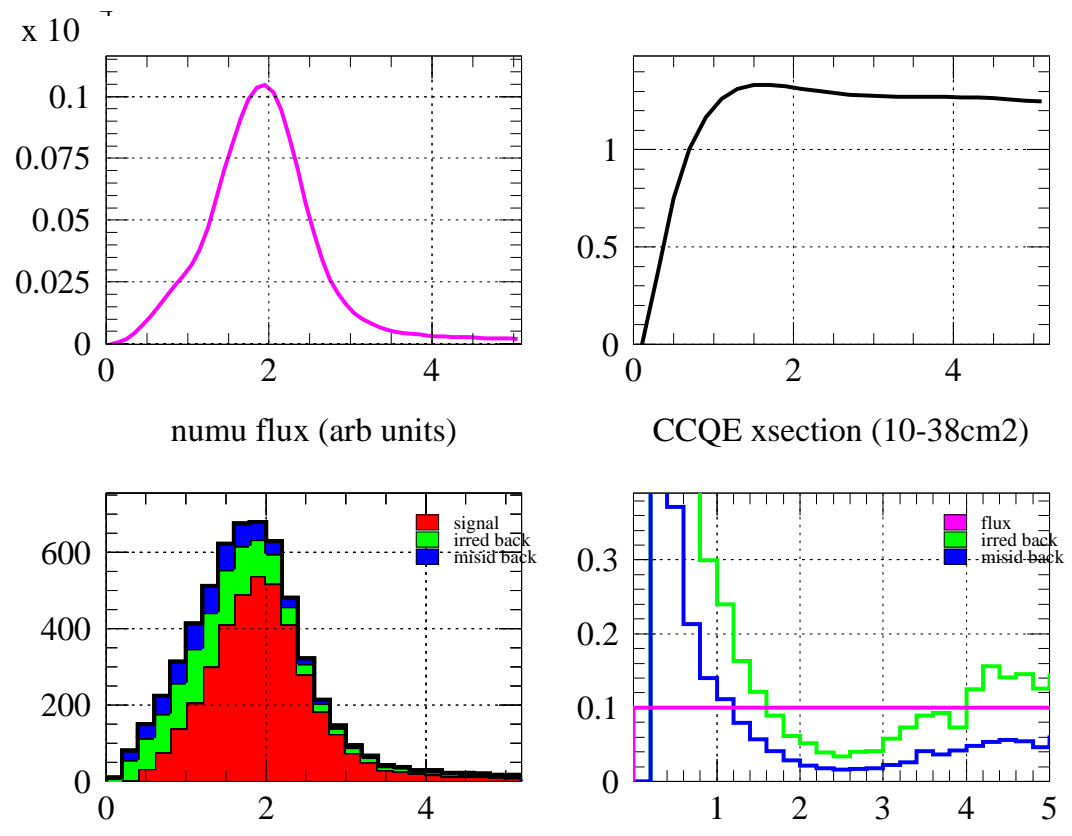

\section{QE events/ton/1E20POT/ebin}
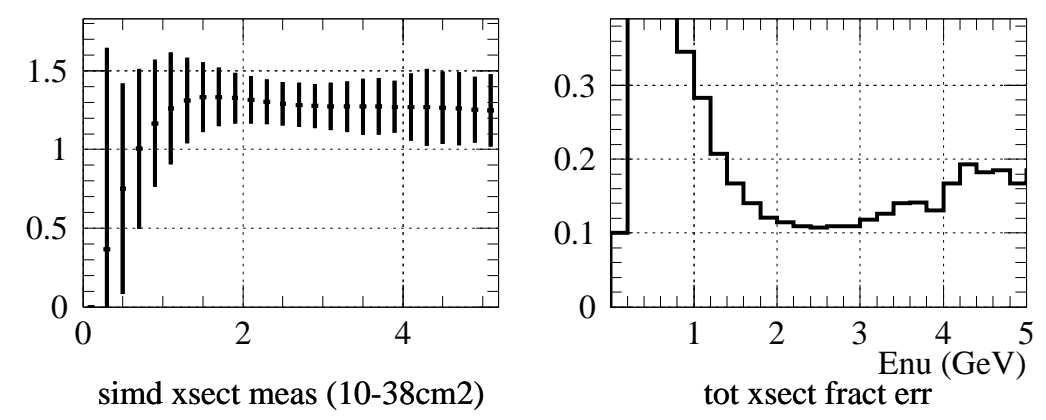

Figure 3: Summary of a NUANCE-based study to estimate systematic errors on a SciNOvA total cross section measurement. The $\nu_{\mu}$ flux and estimated cross section are shown in the top row. The middle left plot shows the estimated number of accepted CCQE events broken down into signal and two $\mathrm{CC} \pi^{+}$background components. The middle right plot shows the fractional systematic error on the cross section due to flux and the two $\mathrm{CC} \pi^{+}$contributions. The bottom left plot shows the estimated cross section with the estimated systematic error bars and the bottom right shows the corresponding cross section fractional errors. The abscissa on all plots is $E_{\nu}$, for the bottom two rows $E_{\nu}$ is that reconstructed with the assumption of quasielastic kinematics. 


\subsubsection{A search for 2-nucleon correlations in CCQE interactions}

Subsequent to the publication of the MiniBooNE CCQE results, a calculation has come to light [9] that successfully reproduces the total cross section as measured by MiniBooNE. This works shows impressive agreement with the MiniBooNE total cross section data (Fig. 4). The main feature of this work is the consideration of multi-nucleon excitations in the carbon nucleus and points out that this produces a sizable increase in the CCQE cross section. This group as well as others (e.g., Ref. [10]) are following this path to see if this model holds up to further comparisons with MiniBooNE data, in particular the MiniBooNE double differential cross sections.

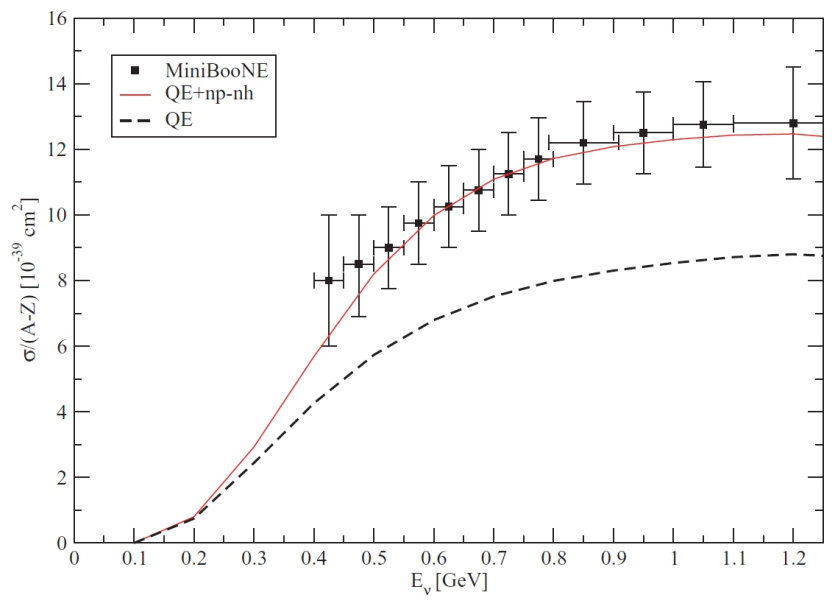

Figure 4: Total cross section for the neutrino CCQE process as measured by MiniBooNE compared with the model of Ref. [9] (solid line) and that model without multinucleon correlations (dashed line). The plot is from Ref. [9].

It may be that multi-nucleon correlations in carbon result in an enhancement of the CCQE cross section as reported by MiniBooNE and modeled in Ref. [9]. Recent results from electron scattering experiments indicate that on average $20 \%$ of the nucleons in carbon are in a 2-nucleon correlated pair (see Ref. [11] and references therein). While it is not completely clear that the nucleon correlations observed in electron scattering are the same physics as the multi-nucleon correlations in Ref. [9], it is an exciting possibility that should be experimentally tested.

SciNOvA can perform this search for 2-nucleon correlations. The energy of the NOvA beam is high enough to provide the energy needed to produce visible correlated nucleon pairs in the final state, yet not so high as to be far from the MiniBooNE peak energy $(800 \mathrm{MeV})$. In addition, the narrow band beam limits the background from $\mathrm{CC} \pi$ channels.

The idea is to search for two protons in the final state of a CCQE interaction that have the characteristics of correlated proton pairs. In practice, we select "CCQE-like" events, events with one identified muon and no observed pions. A typical CCQE-like event in the SciBar detector is shown in Fig. 14. Note the reconstructed recoil proton in this event. We have developed a method, following the techniques demonstrated in electron scattering [11] that will show 2-nucleon correlations if they show themselves in neutrino scattering.

We begin by selecting CCQE-like events, with a well-measured muon with $\boldsymbol{q}=500-900 \mathrm{MeV} / \mathrm{c}$. This corresponds to the $Q^{2}=0.3-0.8 \mathrm{GeV}^{2}$ range where much of the extra cross section is observed in the MiniBooNE experiment. The kinematical variable $\boldsymbol{q}$ is the momentum transfer from the lepton side of the interaction as is defined, as in electron scattering, $\boldsymbol{q}=\boldsymbol{p}_{\nu}-\boldsymbol{p}_{\mu}$. For neutrino scattering, unlike for electron scattering, $\boldsymbol{p}_{\nu}$ is uncertain due to the energy spread of the 
incident neutrino flux. However, assuming quasielastic kinematics, as was done in the MiniBooNE analysis, $\boldsymbol{p}_{\nu}$ may be determined from the muon alone. This cut is not crucial to the analysis but only serves to select candidate CCQE events where 2-nucleon effects may reside.

These candidate events would be searched for two recoil protons that have the expected characteristics of 2-nucleon correlations. Because this is neutrino scattering, where the initial lepton (neutrino) momentum is unknown, our procedure utilizes transverse kinematics where more precise measurements of missing momentum can be made. We have tested it using a NUANCE event generator that was tuned to reproduce the MiniBooNE data. The event selection procedure requires the following criteria:

- Two high-momentum protons in the final state of a CCQE-like event (along with the identified muon). For these protons to be reconstructed in the SciNOvA detector they must have a minimum kinetic energy of approximately $100 \mathrm{MeV}$, which dictates a minimum momentum for each proton of $450 \mathrm{MeV} / \mathrm{c}$.

- The missing transverse momentum calculated from the muon and leading nucleon $\left(p_{T m 1}\right)$ is large compared to the Fermi momentum $\left(p_{F}=220 \mathrm{MeV} / \mathrm{C}\right.$ in carbon).

- The missing transverse momentum when the muon and both protons are considered $\left(p_{T m 2}\right)$ is small compared to $p_{F}$.

- The angle between the proton momentum vectors $(\cos \gamma)$ is near $180^{\circ}$ indicating that they were in a correlated pair with (nearly) equal and opposite momenta.

The characteristics are unique to two nucleon correlation events and are not expected to be satisfied for the backgrounds to this search. The backgrounds are "true" CCQE events and CC $\pi$ events with $\pi$ absorption where multiple nucleons are produced in the final state.

In this NUANCE-based study we modeled the 2-nucleon "signal" events by producing a 2-proton pair with zero center-of-mass momentum and $200 \mathrm{MeV} / \mathrm{c}$ relative momentum in the initial state for $30 \%$ of the CCQE events. The 30\% comes from the simple assumption that the excess cross section as measured by MiniBooNE is associated with 2-nucleon correlations. The model assumed that the momentum transfer in the interaction took place on only one of the protons in the pair. These are conservative model parameters as the pair center-of-mass momentum has been measured to be approximately $140 \mathrm{MeV} / \mathrm{c}$ and the relative momentum is expected to be a wide distribution with tails extending significantly beyond the Fermi momentum [11].

The missing transverse momentum distributions of candidate events in this study are shown in Fig. 5. As can be seen in the figure these distributions are quite distinctive between the signal and backgrounds. The missing transverse momentum cuts $\left(p_{T m 1}>p_{F}, p_{T m 2}<p_{F}\right)$ are then applied. The distribution of the resulting event sample in $\cos \gamma$ is shown in Fig. 6. This plot shows that the backgrounds are both small and readily distinguished when compared to the 2-nucleon signal. A cut requiring $\cos \gamma<-0.5$ is then applied to produce the final sample. The results are summarized in Tab. 2 with the assumption of a 10ton (fiducial) detector with 6E20 protons on target (expected in $1 \mathrm{yr}$ of NOvA running). Note that a large sample of 2-nucleon candidates will be identified if they are present in CCQE-like events. The resulting signal/background ratio is $3: 1$. SciNOvA can make a significant statement on the role of 2-nucleons correlations in the CCQE process.

It should be noted that a full analysis of this would involve not just simple cuts, but comparisons of the various momentum and angle distributions. The distributions offer much physics content and they should be understood on the basis of an underlying physics model even if 2-nucleon correlations do not prove to be substantial in the CCQE neutrino process. The results of this study show that 2-nucleon correlations should show themselves with quite distinctive characteristics. 

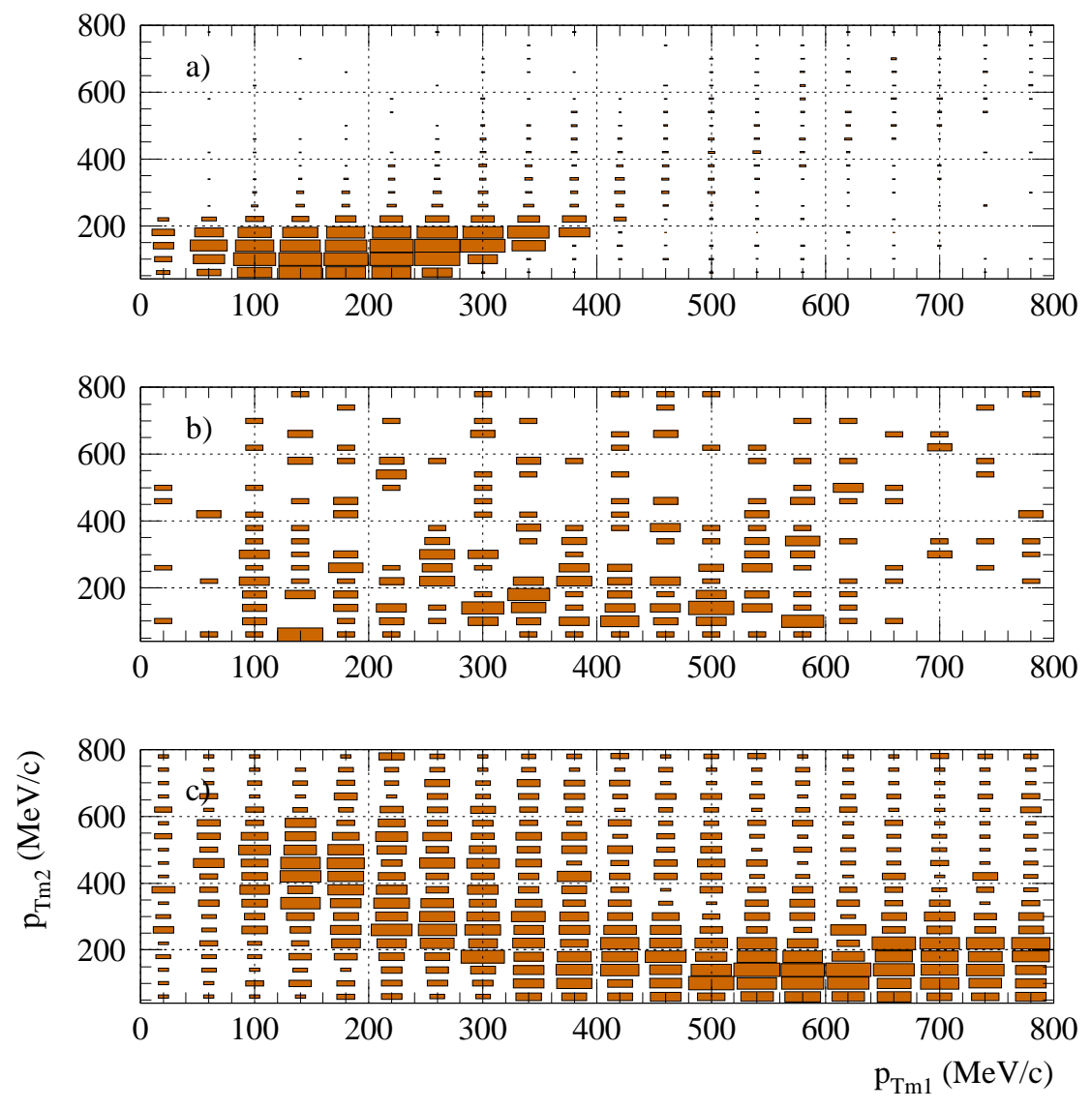

Figure 5: Missing transverse momenta $p_{T m 1}, p_{T m 2}$ 2D distributions for a) 2-nucleon signal, b) CCQE background and, c) QE-like (predominantly $\mathrm{CC} \pi$ ) background events. For events in these plots, the reconstructed momentum transfer was in the range $500<\boldsymbol{q}<900 \mathrm{MeV} / \mathrm{c}$ and 2-protons with $p>450 \mathrm{MeV} / \mathrm{c}$ were required.

\begin{tabular}{ll}
\hline \hline event type & events/10ton/6E20 \\
\hline 2-nucleon signal & 4119 \\
CCQE 1-nucleon background & 65 \\
QElike background & 1320 \\
total background & 1384 \\
\hline
\end{tabular}

Table 2: Accepted events in the 2-nucleon correlation study described in the text. 


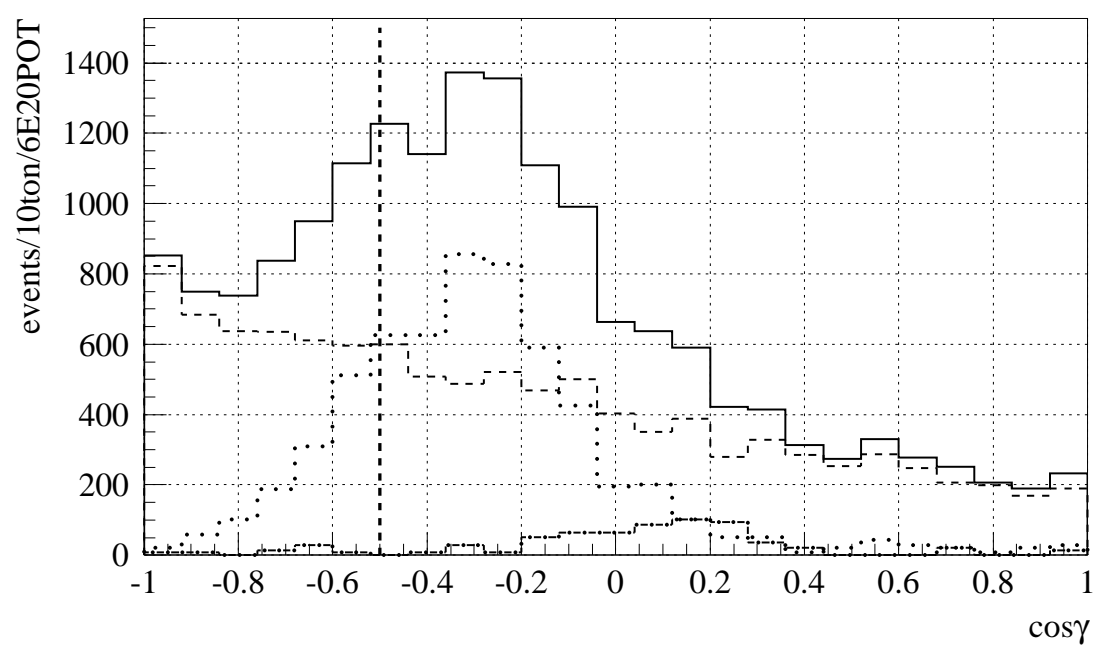

Figure 6: Two-proton opening angle, $\cos \gamma$, distribution for candidate events passing cuts. The lines indicate total events (solid), 2-nucleon signal (dashed), CCQE background (dot-dashed), and QE-like background (dotted). The vertical dashed line indicates the $\cos \gamma<-0.5$ cut applied for the final sample. 


\subsection{Neutral current channels}

Neutrino neutral-current (NC) scattering $(\nu+N \rightarrow \nu+X)$ is important for oscillation experiments as the NC process can contribute substantially to backgrounds for electron neutrino appearance - the primary measurements of the MiniBooNE, T2K, NOvA, and LBNE experiments. This is true because of the large missing energy inherent in NC processes. A narrow-band beam reduces this problem and would allow an associated near experiment to produce NC measurements with reduced uncertainties. In addition to gaining better understanding of $\mathrm{NC}$ processes for oscillation measurements, there are several other physics topics that may be pursued with NC processes.

\subsubsection{Neutral current $\pi^{0}$ production}

Neutral-current $\pi^{0}$ production $\left(\mathrm{NC} \pi^{0}\right)$ in neutrino scattering $\left(\nu+N \rightarrow \nu+\pi^{0}+X\right)$ is a particularly thorny background for $\nu_{e}$-appearance experiments, even though the event rate compared to other neutrino channels is fairly small. MiniBooNE has measured this process in order to obtain a better $\mathrm{NC} \pi^{0}$ background measurement for the oscillation appearance search and in order to investigate coherent $\pi^{0}$ production in the NC process [12]. The MiniBooNE result shows the rate for coherent $\mathrm{NC} \pi^{0}$ production to be substantially lower than predicted. In addition, SciBooNE has measured the coherent $\pi^{0}$ production process [13] in charged-current scattering and has also found it to be lower than predicted. The conclusion from these results is that the coherent production of $\pi^{0}$ on carbon is not well understood theoretically at $E_{\nu} \approx 1 \mathrm{GeV}$ and merits further investigation. The SciBar detector running in a narrow-band beam can contribute to this. As the final state neutrino carries an unknown fraction of the energy in an $\mathrm{NC}$ interaction, the narrow-band neutrino beam is a key feature for understanding the kinematics of NC scattering.

\subsubsection{Neutral current elastic scattering}

A measurement of the neutral current elastic scattering (NCel) process $(\nu+p \rightarrow \nu+p)$ is sensitive to any isoscalar contributions (such as strange quarks) to the spin structure of the nucleon [14]. A measurement of NCel scattering in SciBar in the narrow-band NOvA beam could be sensitive to these contributions. A challenge to this measurement at the NOvA near location is the large low-energy neutron background from neutrino interactions in the rock surrounding the detector. However, it may be that this background could be accurately measured and subtracted from the data. If successful, a neutrino measurement with the SciNOvA experiment could add valuable information to the nucleon spin puzzle.

\subsection{Comparison to wide-band beam measurements}

The MINERvA experiment is currently running at Fermilab with an impressive array of neutrino and nuclear physics goals. SciNOvA will complement this program by focusing on utilizing the NOvA narrow-band beam to measure the CCQE and $\mathrm{NC} \pi^{0}$ processes at lower energies $(\approx 2 \mathrm{GeV})$ with greate precision than is possible with a higher-energy wide-band beam.

We have estimated the systematic errors on a total CCQE cross section measurement an experiment in a wide band beam in both the low- and medium-energy neutrino beam configurations as expected for MINERvA. The method used was the same as that described in Sec. 2.1 and presented in Fig. 3. It involved boot-strapping the systematic errors from the MiniBooNE CCQE measurement and it assumed that statistical errors are negligible. These results are summarized in Figs. 7 and 8 . 
The main result of this study is that the systematic error on the total cross section for a broadband beam measurement such as MINERvA will be too large at $2 \mathrm{GeV}$ to definitively resolve the discrepancy between the MiniBooNE and NOMAD measurements (Fig. 2). The main reason for this is the large $(25 \%)$ uncertainty in $\pi$ absorption combined with the large "feed down" of CC $\pi$ events from higher neutrino energy. It may be that the pion loss in the detector may be reduced from the value of $10 \%$ that came from MiniBooNE analysis. However, the dominant contribution to the error is from the uncertainty on pions that are absorbed in the nucleus which can not be reduced with improvements to pion identification.

This study indicates that the fractional error on the total cross section will be approximately $25 \%$ and $35 \%$ in the low- and medium-energy beam configurations respectively. This is not sufficient to test the MiniBooNE result which shows an approximately $30 \%$ enhancement of the CCQE cross section over that expected from a canonical model with the world-average $M_{A}$ value, a model that adequately describes the NOMAD results. It should be pointed out that these estimates show cross section errors reducing to those of the flux normalization closer to the peak of the flux. Measurements at these higher energies will be quite valuable in understanding the differences between the MiniBooNE and NOMAD results. However, it is likely that the MINERvA measurements alone will not be inadequate to understand the discrepancy completely.

In addition, a measurement of the $\mathrm{NC} \pi^{0}$ process with SciNOvA as compared to MINERvA is more directly relevant to the NOvA oscillation program since SciNOvA will measure $\mathrm{NC} \pi^{0}$ from the same beam as the NOvA near detector. This will allow for the cancellation of many systematic errors in data driven techniques (Sec. 2.4). These errors will be substantial in extrapolating $\mathrm{NC} \pi^{0}$ cross sections measured in MINERvA to NOvA. 

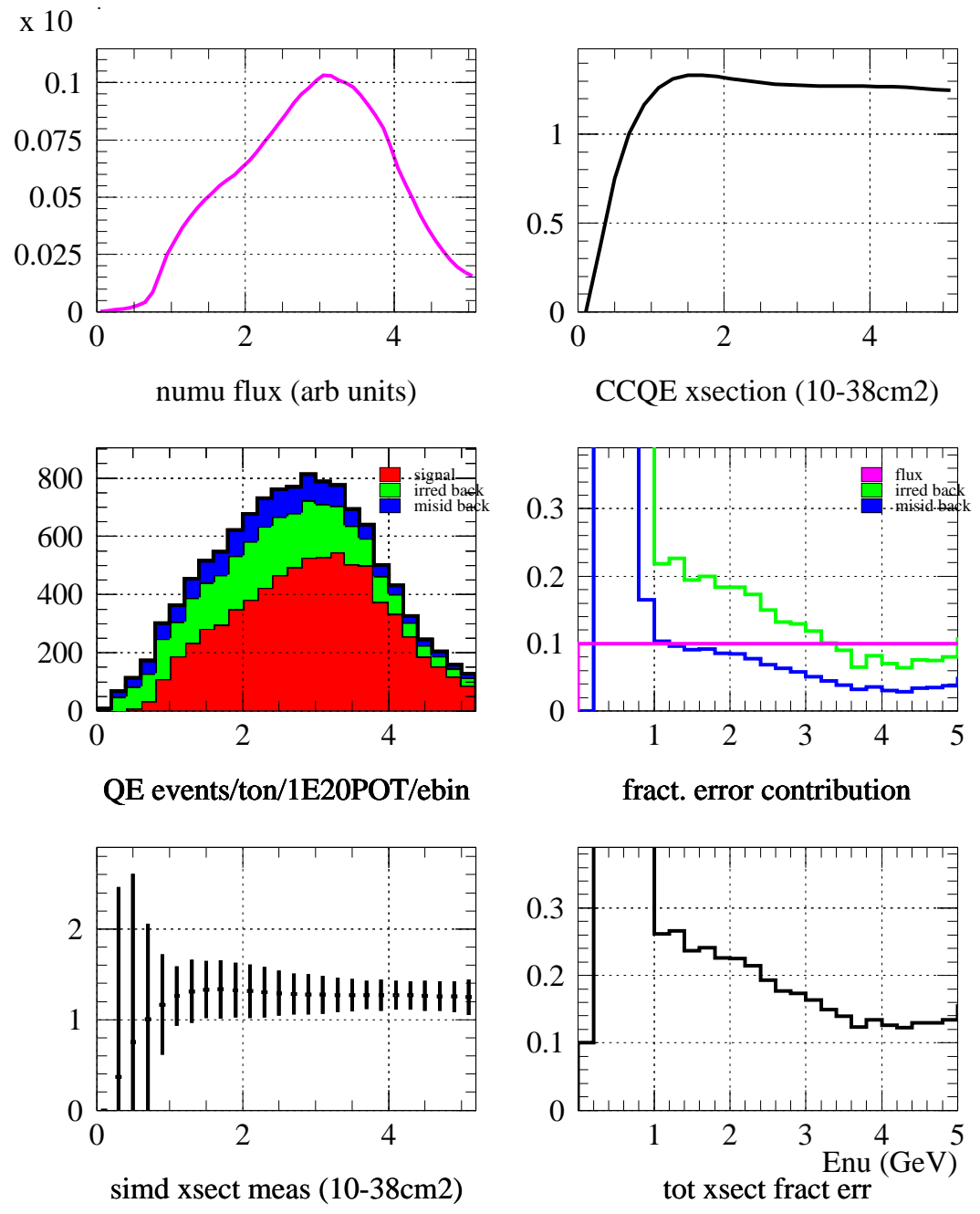

Figure 7: Summary of a NUANCE-based study to estimate systematic errors of cross section measurement in a wide-band low-energy neutrino beam configuration. The $\nu_{\mu}$ flux and estimated cross section are shown in the top row. The middle left plot shows the estimated number of accepted CCQE events broken down into signal and two $\mathrm{CC} \pi^{+}$background components. The middle right plot shows the fractional systematic error on the cross section due to flux and the two $\mathrm{CC} \pi^{+}$contributions. The bottom left plot shows the estimated cross section with the estimated systematic error bars and the bottom right shows the corresponding cross section fractional errors. The abscissa on all plots is $E_{\nu}$, for the bottom two rows $E_{\nu}$ is that reconstructed with the assumption of quasielastic kinematics. 

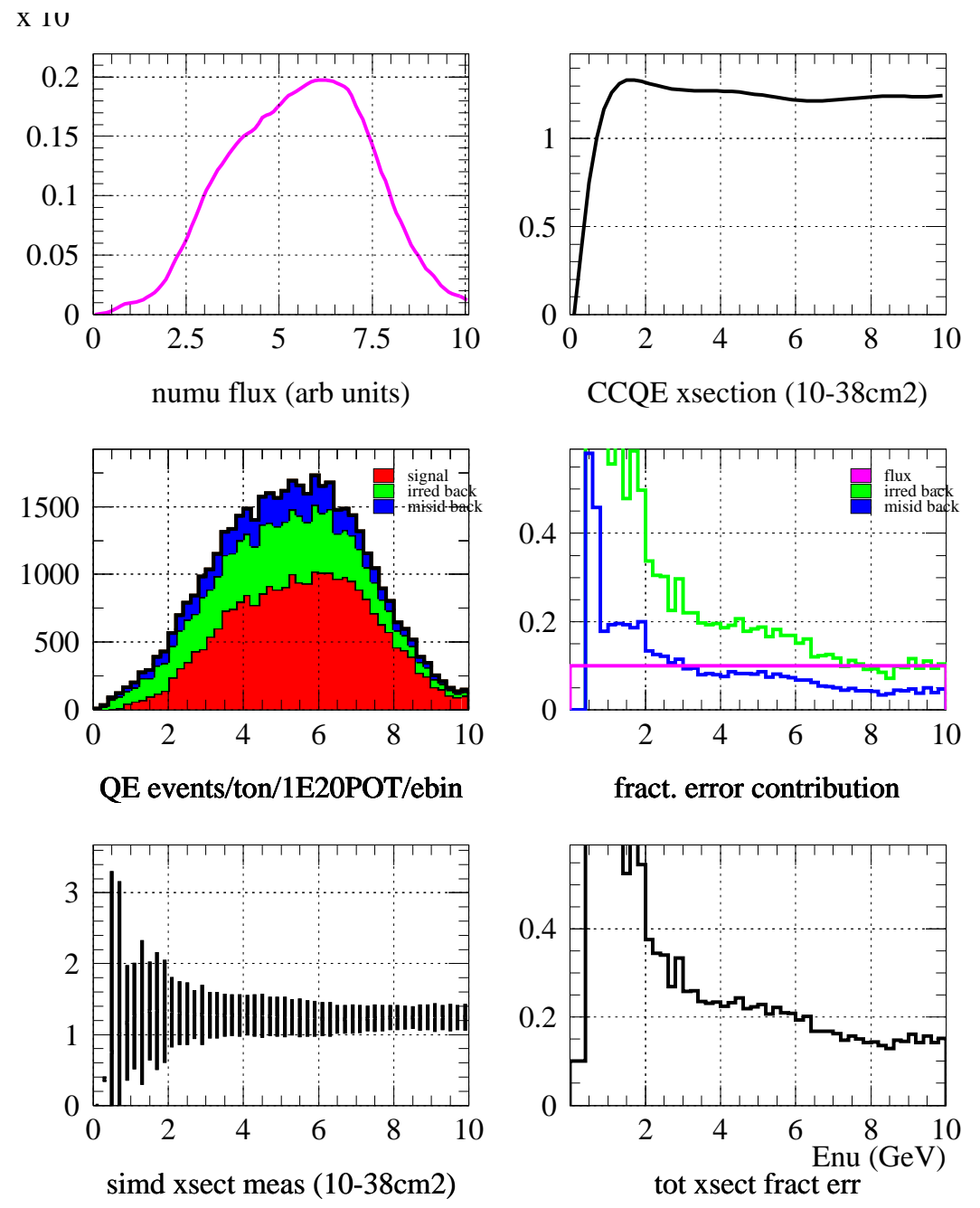

Figure 8: Summary of a NUANCE-based study to estimate systematic errors of cross section measurement in a wide-band medium-energy neutrino beam configuration. Explanation of plots is the same as in Fig. 7. 


\subsection{Application to the NOvA experiment}

The NOvA experiment will search for $\nu_{\mu} \rightarrow \nu_{e}$ and $\bar{\nu}_{\mu} \rightarrow \bar{\nu}_{e}$ oscillations using the NuMI neutrino beam at Fermilab and two detectors located in Ash River, MN at a distance of $810 \mathrm{~km}$ from the neutrino source and underground at Fermilab at a distance of $1 \mathrm{~km}$ from the neutrino source. The experiment will be sensitive to values of the mixing parameter $\sin ^{2} 2 \theta_{13}$ down to 0.01 at $90 \%$ C.L. Should $\nu_{\mu} \rightarrow \nu_{e}$ and $\bar{\nu}_{\mu} \rightarrow \bar{\nu}_{e}$ oscillations be observed, NOvA will use a comparison of the oscillation rates to resolve the neutrino mass hierarchy and begin the study of the CP-violating phase $\delta$. These questions have been highlighted by many physics advisory committees (for example [15]) as among the most important outstanding questions in neutrino and particle physics. A possible NOvA result from $\nu_{\mu} \rightarrow \nu_{e}$ and $\bar{\nu}_{\mu} \rightarrow \bar{\nu}_{e}$ oscillations is shown in Fig. 9 demonstrating NOvA's ability to measure the mixing angle $\theta_{13}$, resolve the neutrino mass hierarchy, and reducing the possible range of the CP violating phase $\delta$ by half. The NOvA far detector laboratory is nearing completion with occupancy of the building expected in spring of 2011 and a prototype detector will begin operation this year. The completed experiment will begin taking data in 2013.

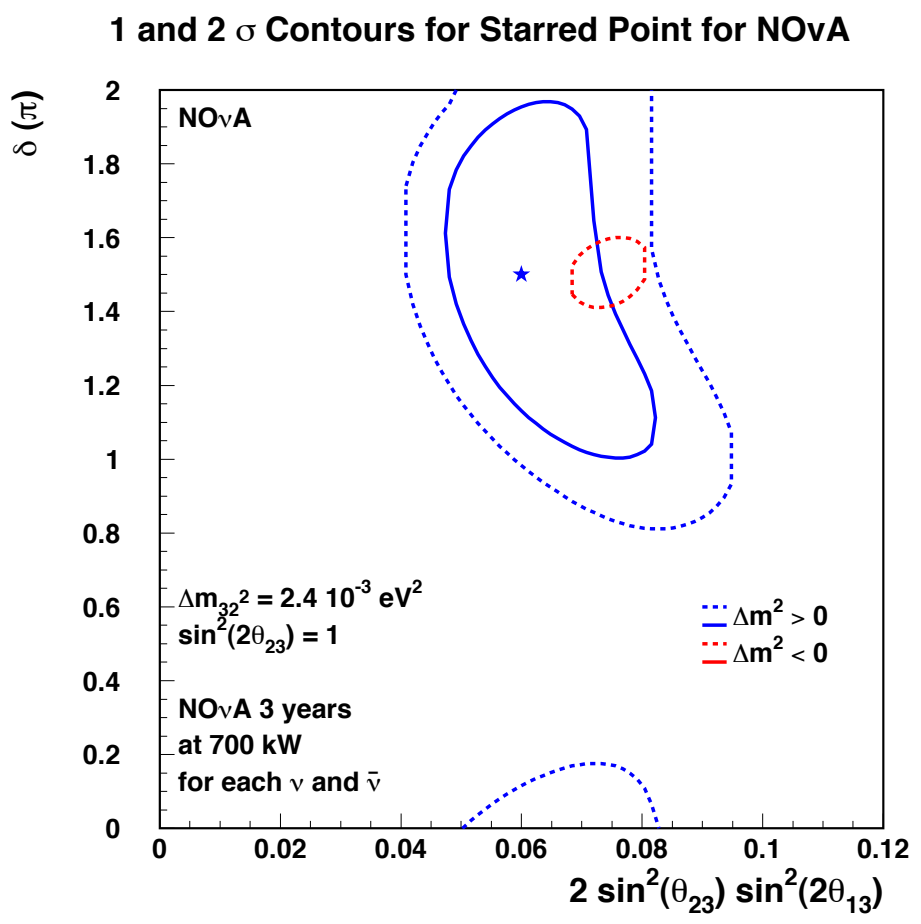

Figure 9: Simulated result from the NOvA experiment. The small red region indicates the allowed region assuming an inverted hierarchy. As this region appears only at 2-sigma, the hierarchy question is resolved in favor of the normal hierarchy.

The NOvA detectors are made from hollow PVC extrusions which contain liquid scintillator in rectangular cells measuring $6.2 \mathrm{~cm}$ (longitudinal) $\times 3.9 \mathrm{~cm}$ (transverse). These correspond to $14 \%$ of a radiation length and $37 \%$ of a Moliere radius respectively. Electron-neutrino interactions are identified by searching for neutrino interactions which contain a prong with a longitudinal and 
transverse profile consistent with an electro-magnetic shower. The primary backgrounds to this search originate from neutral-current interactions which contain electro-magnetic showers from $\pi^{0} \rightarrow \gamma \gamma$ and the intrinsic electron-neutrino component of the neutrino beam which result from muon and kaon decays. The NOvA detectors identify electron-neutrino charged-current (CC) events with an efficiency of 35\% while accepting neutral-current (NC) backgrounds and muon-neutrino $\mathrm{CC}$ events into the signal sample with $0.4 \%$ and $0.1 \%$ probabilities respectively.
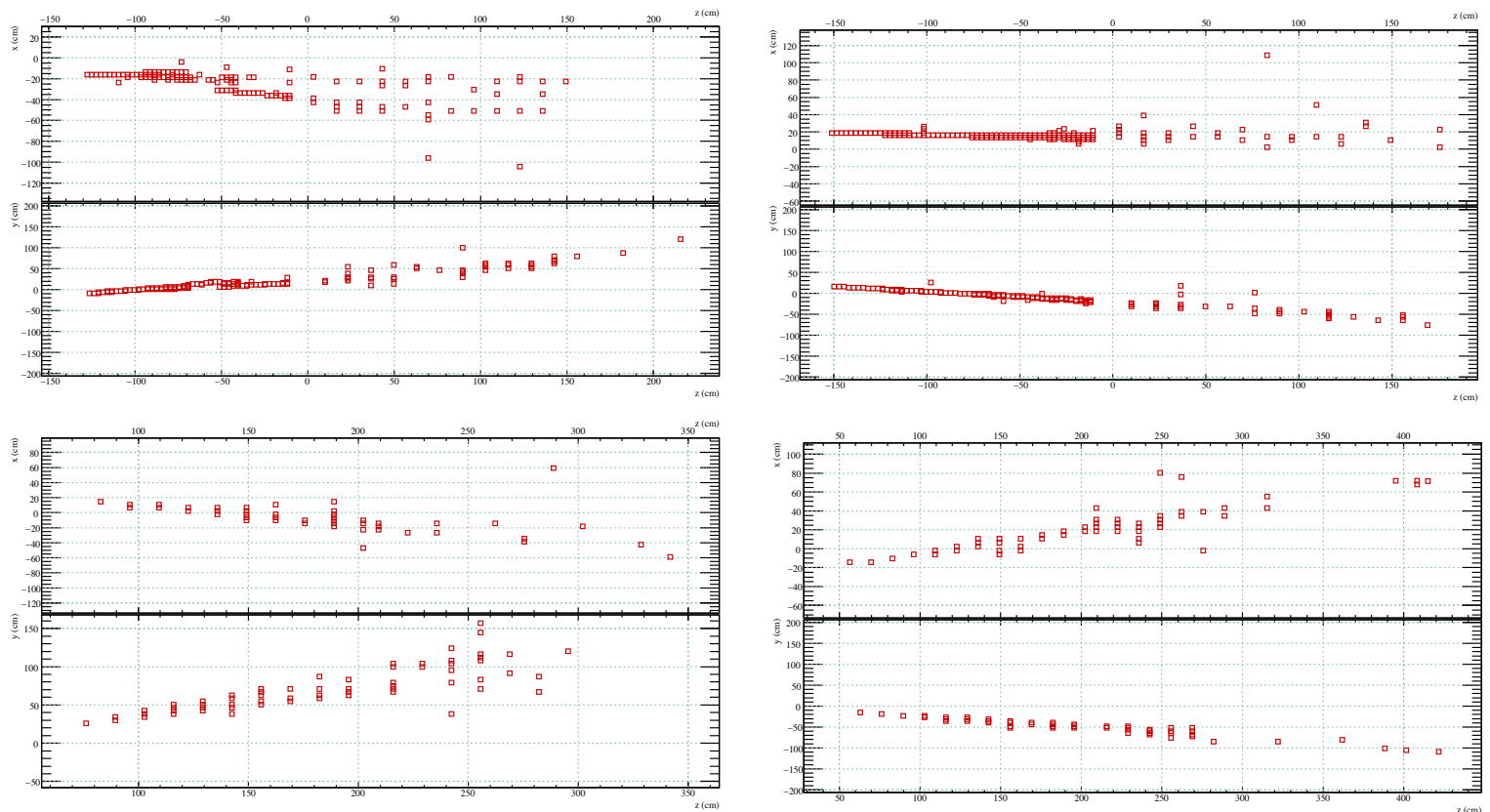

Figure 10: Sample event displays in the SciBar (top) and NOvA (bottom) detectors. The left two panels show the response to a single $2 \mathrm{GeV} \pi^{0}$. The right two panels show the response to a single $2 \mathrm{GeV}$ electron.

The background rejection factors for the experiment must not only be large, but they must also be well known to avoid having the sensitivity of the experiment degraded by uncertainties on the backgrounds. As the rejection factors are large, they can be quite difficult to estimate as the background events which leak into the signal region tend to be on the tails of all the distributions used to separate signal and background events. We note that there is still considerable skepticism of several reports of electron neutrino appearance by oscillation experiments (e.g., LSND [16] and MiniBooNE [17] due to questions about the background estimates.

NOvA will use several data-driven techniques which have been applied by previous appearance experiments to verify its signal efficiency and background rejection estimates. These include analysis of data recorded with a near detector, analysis of $\nu_{\mu}$-CC events with the muon track removed, and analysis of $\nu_{\mu}$-CC events where the muon is replaced with a simulated electron shower. The SciBar detector can contribute to all of these and its finer granularity will enhance the performance of each of these techniques. For example, techniques which fit and remove muons from neutrino events are more easily applied to a fine grained detector where there is little light sharing among the detector hits. Likewise, $\pi^{0}$ production rates measured in the SciNOvA detector will also be valuable inputs to the background simulations for the NOvA detector. Beyond enhancing existing techniques for understanding signal and background events, the addition of the SciNOvA detector will make an additional powerful cross-check of the NOvA detector performance possible by providing an 
independent and more efficient signal/background determination which can be compared to the determination made by the NOvA detector. This principle of this technique is outlined below.

\subsection{1 "Double scan" by event resampling}

The proposed technique to cross-check the signal and background efficiencies in the NOvA detector is similar in concept to techniques used in the analysis of bubble chamber data. Photographs of bubble chamber events were double scanned to search for signal events. The two scanners in general would not have the same finding efficiency $\left(\epsilon_{1}\right.$ and $\left.\epsilon_{2}\right)$ and would record different number of events $N_{1}$ and $N_{2}$ as signal events. The samples from the two scanners would have $N_{12}$ events in common. From this information it is possible to compute the efficiencies of the two scanners and the unknown true signal count $S$ in the original event sample:

$$
\epsilon_{1}=\frac{N_{12}}{N_{2}}, \quad \epsilon_{2}=\frac{N_{12}}{N_{1}}, \quad S=\frac{N_{1} N_{2}}{N_{12}}
$$

Using the finer grained SciBar detector, one can use a similar technique by analyzing the event topologies as recorded by the SciBar detector and as they would have been recorded by the NOvA detector.

\begin{tabular}{|c|c|c|c|c|c|}
\hline \multirow{3}{*}{$\mathrm{NOvA}$} & \multicolumn{4}{|c|}{ "Segmentation } & \multirow[t]{2}{*}{ "Composition by weight } \\
\hline & \multicolumn{2}{|c|}{ Longitudinal } & \multicolumn{2}{|c|}{ Transverse } & \\
\hline & $6.2 \mathrm{~cm}$ & $14 \% X_{0}$ & $3.9 \mathrm{~cm}$ & $37 \% R_{M}$ & $\begin{array}{l}70.4 \% \mathrm{C}, 14.5 \% \mathrm{Cl}, 9.7 \% \mathrm{H} \\
2.7 \% \mathrm{Ti}, 2.2 \% \mathrm{O}, \text { others }<1 \%\end{array}$ \\
\hline SciBar & $1.3 \mathrm{~cm}$ & $3 \% X_{0}$ & $2.5 \mathrm{~cm}$ & $30 \% R_{M}$ & $\begin{array}{l}92.2 \% \mathrm{C}, 7.8 \% \mathrm{H} \\
\text { others }<1 \%\end{array}$ \\
\hline
\end{tabular}

Table 3: Comparisons of the constructions of the NOvA and SciBar detectors.

Table 3 compares the segmentation and composition of the NOvA and SciBar detectors. As the detectors would be placed at the same location in the NuMI beam they would be exposed to an identical neutrino flux. Further, as both detectors are largely composed of hydrocarbons, the neutrino cross-sections in both detectors would be nearly identical. Thus, we would collect two samples of neutrino interactions whose underlying physics distributions are nearly identical but differ in the resolution of the detector that recorded them.

To apply this "double scan" technique, the events recorded by the SciBar detector would be analyzed and classified as either signal or background. As the SciBar is more highly segmented than the NOvA detector one could then combine the signals from adjacent scintillator bars (sharing charge across multiple cells where the detector boundaries do not meet exactly) until the event topology resembled that of the coarser grained NOvA detector. Each event in the sample would then be reexamined using the NOvA analysis and reclassified as signal or background. The procedure is outlined schematically in Figure 11. This "double scan" would sort the $N$ events in the sample into four categories: $N_{s s}$ events where both detectors classify the events as signal, $N_{b b}$ events where both detectors find background, and $N_{s b}$ and $N_{b s}$ where one detector finds signal and the other finds background. These are related to the signal efficiencies of the two detectors $\epsilon_{S B}$ and $\epsilon_{N}$ (where 


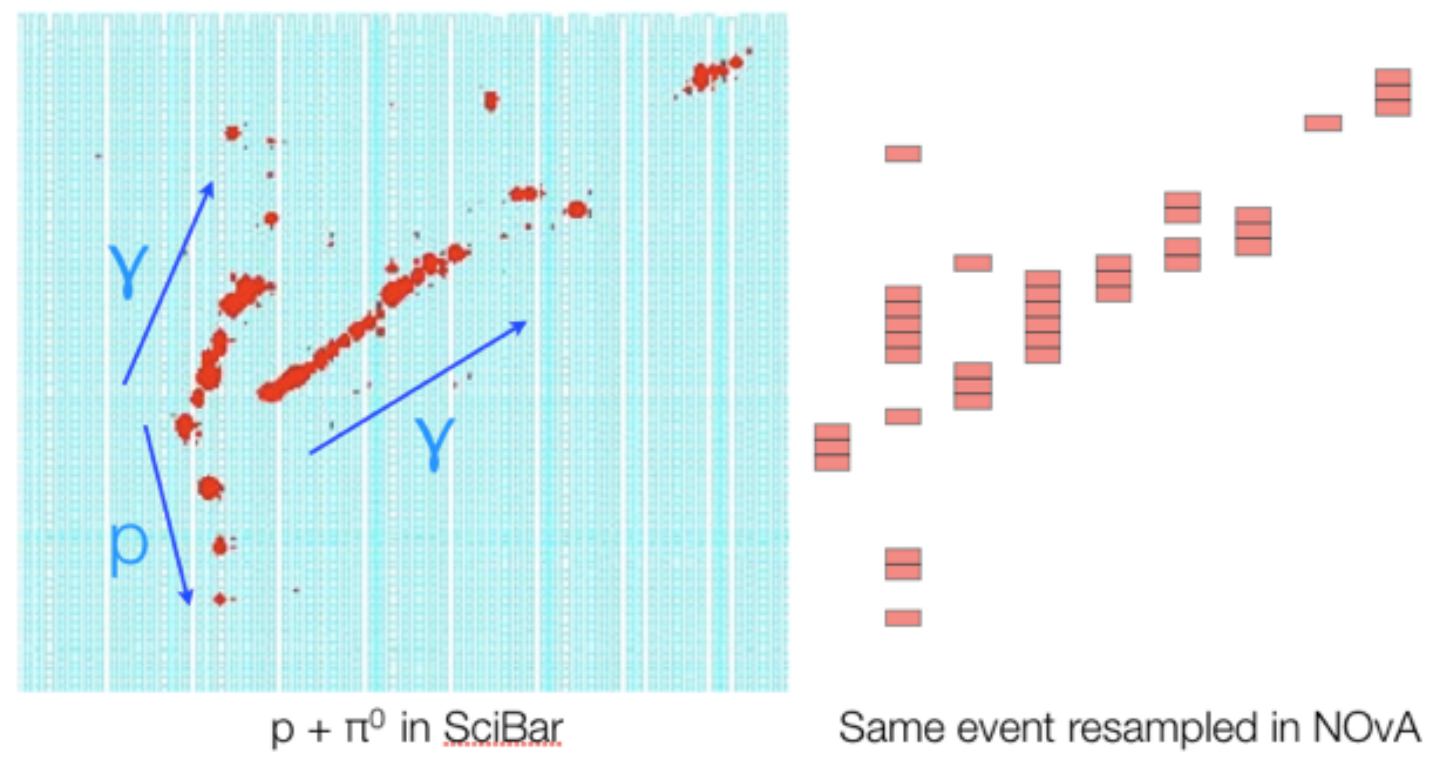

Figure 11: Demonstration of the resampling technique. Left panel shows an actual event recorded by the SciBar detector in the Booster neutrino beamline as part of the SciBooNE experiment; presumably $\nu_{\mu}+p \rightarrow \nu_{\mu}+p+\pi^{0}$. The right panel shows how this same event would appear in the NOvA detector.

$S B \equiv \mathrm{SciBar}$ and $N \equiv \mathrm{NOvA}$ ) and the background mis-ID probabilities $\gamma_{S B}$ and $\gamma_{N}$ :

$$
\begin{aligned}
& N_{s s}=\epsilon_{S B} \epsilon_{N} S+\gamma_{S B} \gamma_{N} B \\
& N_{s b}=\epsilon_{S B}\left(1-\epsilon_{N}\right) S+\gamma_{S B}\left(1-\gamma_{N}\right) B \\
& N_{b s}=\left(1-\epsilon_{S B}\right) \epsilon_{N} S+\left(1-\gamma_{S B}\right) \gamma_{N} B \\
& N_{b b}=\left(1-\epsilon_{S B}\right)\left(1-\epsilon_{N}\right) S+\left(1-\gamma_{S B}\right)\left(1-\gamma_{N}\right) B .
\end{aligned}
$$

Due to the constraints $N_{s s}+N_{s b}+N_{b s}+N_{b b}=S+B=N$, only three of these equations are linearly independent and a complete solution for the five unknowns $\epsilon_{S B}, \epsilon_{N}, \gamma_{S B}, \gamma_{N}$, and $S$ cannot be obtained. However, if estimates of any two parameters can be made, any of the other three can be calculated. For example, if we have reliable estimates for the efficiency of the SciBar detector $\epsilon_{S B}$ and the number of signal events in the sample $S$ then the maximum likelihood solution for the remaining parameters (to first order in the $\gamma_{\mathrm{s}}$ ) is

$$
\begin{aligned}
\epsilon_{N} & =\frac{1}{\epsilon_{S B}} \frac{N_{s s}}{S} \\
\gamma_{N} & =\frac{\epsilon_{S B}\left(N_{s s}+N_{b s}\right)-N_{s s}}{\epsilon_{S B}(N-S)} \\
\gamma_{S B} & =\frac{N_{s b}+N_{s s}-\epsilon_{S B} S}{N-S} .
\end{aligned}
$$

Likewise, estimates for any two parameters yields predictions for the remaining three. As the experiment will have techniques to produce estimates for all five parameters, one could cycle through the permutations and demand consistency in each case as confirmation that the efficiencies, misidentification probabilities, and signal rates have been correctly estimated. In practice, this may not be done algebraically as shown here, but through iterative tuning of a Monte Carlo simulation to fit the data in each of the four categories. 


\begin{tabular}{llllll} 
& $N_{s s}$ & $N_{s b}$ & $N_{b s}$ & $N_{b b}$ & $\chi^{2}$ \\
\hline Nominal & 15500 & 50300 & 66600 & 10867600 & - \\
\hline$\gamma_{N}$ higher by 10\% & - & - & +4300 & -4300 & 279 \\
$\gamma_{N}$ and $\gamma_{S B}$ higher by 10\% & - & +2200 & +4300 & -6500 & 371 \\
$B$ higher by 10\% & -1500 & -2800 & -2300 & +6600 & 403 \\
\hline
\end{tabular}

Table 4: Summaries of the event counts for one year's SciNOvA data in each of the four classification categories. Changes in the counts for three different possible systematic differences from the nominal case are shown and the $\chi^{2}$ significance of the differences appears in the last column.

\subsubsection{Numerical example}

Sample event displays for $2 \mathrm{GeV}$ electrons and $\pi_{0} \mathrm{~s}$ are shown in Figure 10. Based on blind hand scan of mixed electron and $\pi^{0}$ events in the NOvA and SciBar detectors it was found that the efficiency of the SciBar detector is roughly $10 \%$ higher than the NOvA detector and that the SciBar detector rejects background at twice the rate of the NOvA detector. These give $\epsilon_{N}=0.35, \epsilon_{S B}=0.40$, $\gamma_{N}=0.004$, and $\gamma_{S B}=0.002$. In one year of NOvA operation we expect to record $1.1 \mathrm{M}$ events in the SciBar detector. Of these $1 \%(11,000)$ will be electron neutrino charged-current events the signal topology for the NOvA experiment. Table 4 shows the expected count rates in each of the four classification categories for the nominal case, and three cases where either the background rejection capabilities of the detectors is worse than estimated or the backgrounds in the underlying sample are larger than expected. Note that these cases are distinguishable as they affect each of the four samples differently. The sensitivity of this technique is such that a $1.8 \%$ change of the NOvA background mis-ID probability from 0.0040 to 0.00407 results in a $3 \sigma$ discrepancy in the event counts. Likewise, changes in $\epsilon_{N}, \epsilon_{S B}, \gamma_{S B}$, and $S$ of $1.8 \%, 1.6 \%, 3.1 \%$, and $1.5 \%$ respectively result in $3 \sigma$ deviations. Thus one would expect this technique to find any miscalculations of the signal and background efficiencies as large as a few percent. This method is a powerful cross-check of the detector capabilities that uses the neutrino data itself and has very little reliance on Monte Carlo simulation and can protect the experiment from unexpected misestimates of background rates. 


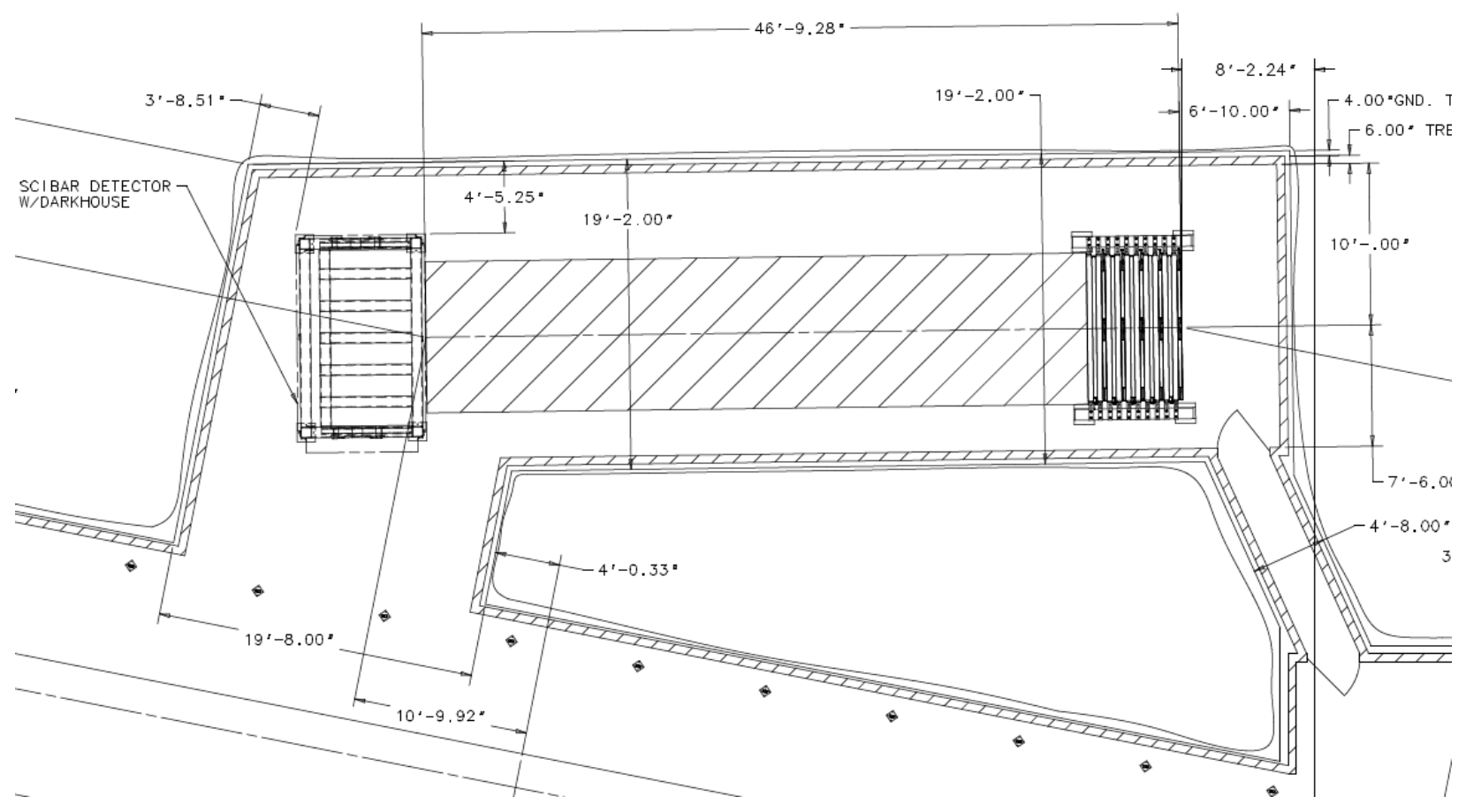

Figure 12: Plan view of the SciBar detector located in the NOvA near tunnel. The SciBar detector will fit into the planned NOvA area without out modifcations to the cavern excavation.

\section{Experiment}

\subsection{Overview}

The SciNOvA experiment will use a highly-segmented solid scintillator "SciBar" detector located directly upstream of the NOvA near detector in the underground hall on site at FNAL as shown in Fig. 12. The $\approx 15 \mathrm{k}$ channel SciBar detector will be constructed at FNAL and will use the same scintillator extrusions as for the original SciBar detector [18] that was used first in the K2K near experiment in Japan 2003-2004 and then in the SciBooNE experiment [19] that ran 2007-2008 on the Booster Neutrino beamline at Fermilab. We would construct a new SciBar detector using the manufacturing facility at Fermilab, but would use the same extrusion size as for the original. The light from the scintillator extrusions is collected via wavelength shifting (WLS) fibers and routed to 64-anode multianode photomultiplier tubes (MAPMTs). A new readout system will be built based on an existing design developed for the "scibath" detector readout [20].

\section{$3.2 \quad$ Scibar detector}

The new SciBar detector that we are proposing to build will be of the same design as the original SciBar detector [18] built for the K2K near detector and shown in Fig. 13. The electromagnetic calorimeter of the original detector will not be used for our application. The detector consists of 14848 extruded $1.3 \times 2.5 \times 290 \mathrm{~cm}^{3}$ scintillator strips arranged in 64 layers. Each layer consists of an $X$ and $Y$ plane, each containing 116 strips. The total active volume of the scintillator is $2.9 \times 2.9 \times 1.7 \mathrm{~m}^{3}$ with total mass of 15 tons. Each strip contains an embedded $1.5 \mathrm{~mm}$ diameter 
wavelength-shifting fiber which are routed to 64-fiber "cookies" which will mate to the MAPMT readout system.

This detector is of proven design and has excellent tracking capabilities as can be seen in the SciBooNE CCQE candidate event display of Fig. 14. The pixelation will allow precise measurement of both the muon and recoil proton (down to approximately $100 \mathrm{MeV}$ kinetic energy) in CCQE events.

This new SciBar detector will require new FNAL-NICADD extruded scintillator [21] of cross section $1.3 \times 2.5 \mathrm{~cm}^{2}$ and length $290 \mathrm{~cm}$ exactly as was manufactured for the original SciBar detector. The technique has been refined with the manufacture of batches for other experiments such as MINERvA [22]. The strips are made of polystyrene doped with PPO and POPOP and are co-extruded with a $\mathrm{TiO}_{2}$ reflective coating $0.25 \mathrm{~mm}$ thick. The peak emission wavelength convoluted with the plastic transmittance for this scintillator peaks at $420 \mathrm{~nm}$. A $1.8 \mathrm{~mm}$ hole runs the length of strip, centered in cross section, to contain the $1.5 \mathrm{~mm}$ diameter WLS readout fiber.

The WLS fibers will be inserted into the scintillator extrusions during the detector assembly process. A blue to green WLS fiber will be used with absorption/emission wavelengths around $430 / 480 \mathrm{~nm}$, respectively. No glue or index matching gel will be used as light collection is sufficient without and quality control of glue or gel is problematic. The fibers will be read out on only one end. The other end of the fiber will be cut at the same length as the scintillator bars. That fiber end may be used to insert a calibration light source but that is not part of the baseline design which will use charged-particle tracks for calibration. On the readout end, the fibers will extend beyond the end of scintillator bars by approximately $50 \mathrm{~cm}$ so they may be gathered into a 64 -fiber bundle and cast into a PMT-interface "cookie".

The fiber cookies are designed to interface with a 64-anode MAPMT with $2 \mathrm{~mm}$ square pixels arranged in an $8 \times 8$ array. The fibers are inserted into a plastic cookie, potted with a two-part machinable epoxy and diamond fly cut so that an optically smooth surface touches the MAPMT glass. The cookie has carefully machined mating surfaces so that the fibers align well with the PMT pixels. The PMTs are housed in a complementary structure to mate with the fiber cookies. The alignment of the MAPMT pixels are not specified with respect to the outer case of the MAPMT housing. Instead, optical fiducial marks are provided on the MAPMT so that the pixels may be carefully aligned with the PMT housing. The PMT housing mates with the fiber cookie to assure pixel/PMT alignment to approximately $0.05 \mathrm{~mm}$, sufficient to minimize optical cross talk between fibers and MAPMT pixels.

\subsection{Readout system}

The 14848 individual readout fibers require 232 64-anode PMTs with associated electronics to provide the required high-voltage for the tubes and to digitize the signals in response to light pulses from the scintillator bars. We propose to use an integrated MAPMT/readout boards developed at Indiana University. These boards were conceived and designed for the FINeSSE experiment [14] in order to provide a low channel-cost readout system for MAPMTs. A 12-PMT system has been built and is running at IU. This system could be replicated in order to provide the readout for the 232 PMTs required for the SciBar readout.

\subsubsection{PMTs}

We anticipate using Hamamatsu H8804 (or equivalent) MAPMTs. This model has been used by many experiments with WLS fiber readout. It has a bialkali cathode yielding a quantum efficiency of $\approx 12 \%$ at the WLS fiber emission wavelength. They run at about $800 \mathrm{~V}$ providing a gain of 


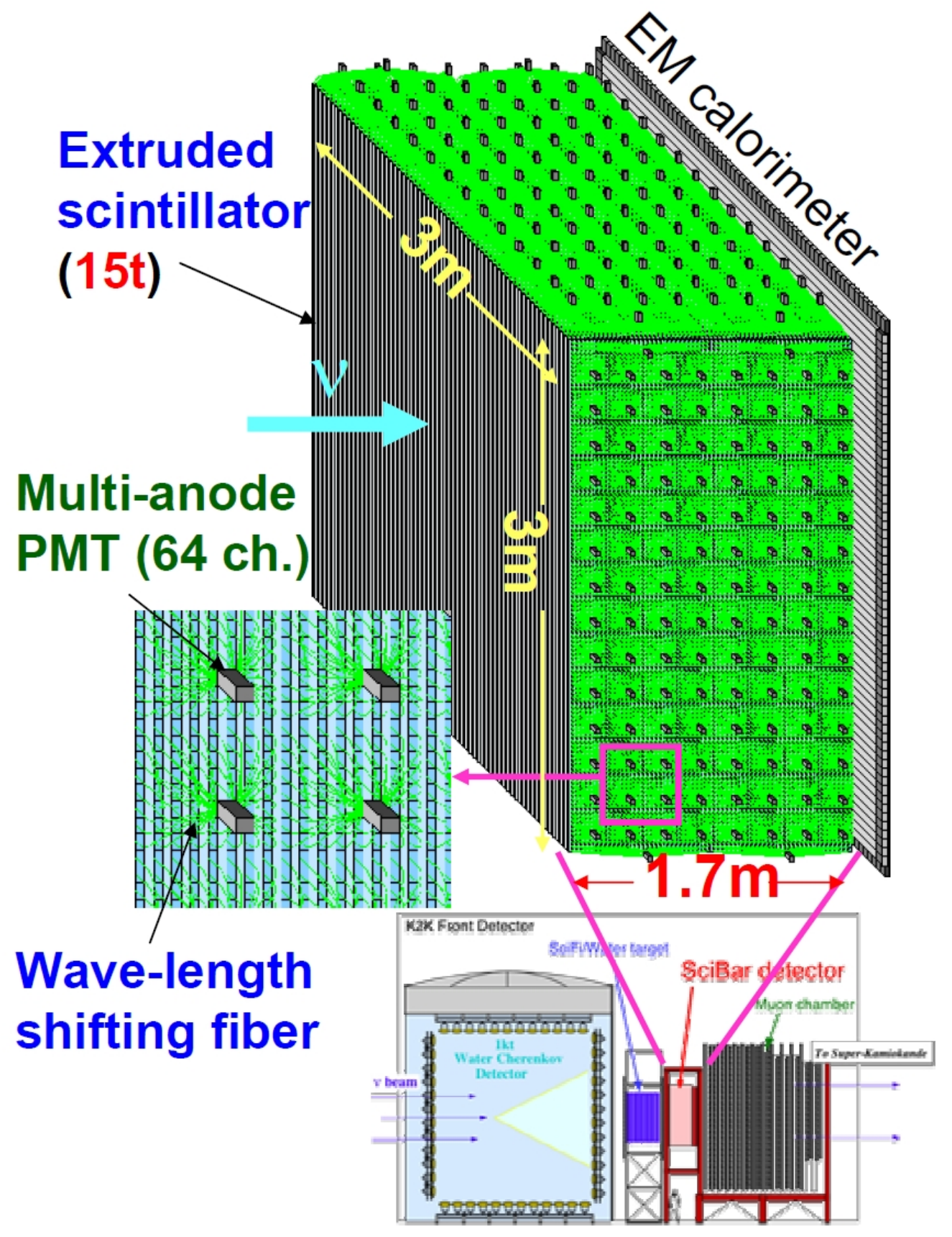

Figure 13: Schematic drawing of the original SciBar detector as employed the K2K near experiment [18]. The EM calorimeter will not be used in SciNOvA 


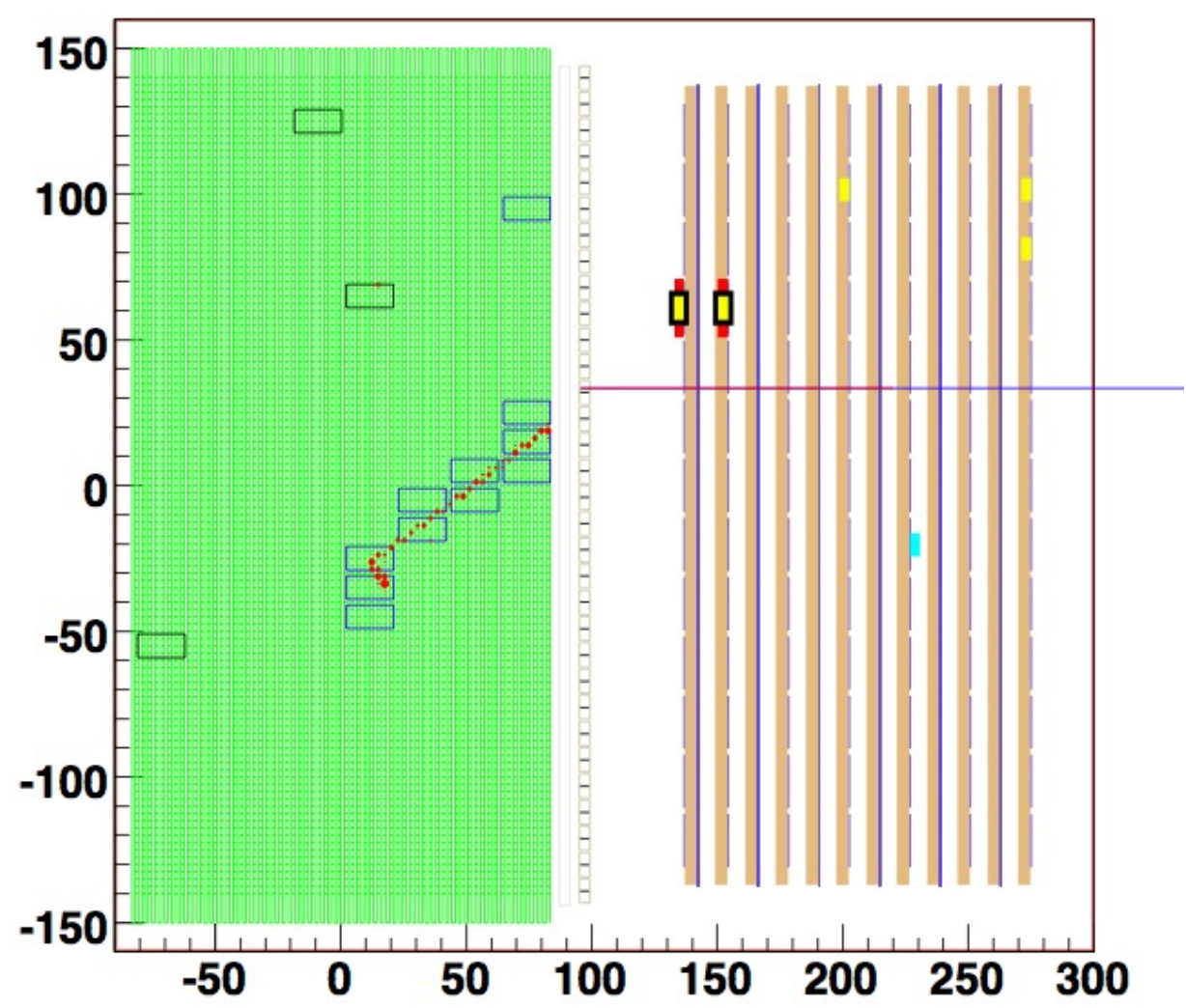

Figure 14: CCQE event display from SciBooNE. The SciBar detector that is proposed for SciNOvA is the green area to the left side where the small red dots show hits in individual bars. The larger blue rectangles show regions of the detector which share common time readout. The brown layers to the right indicate the SciBooNE muon range detector which will not be used in SciNOvA. The NOvA near detector will accept and range out muons that escape the SciBar detector. A reconstructed CCQE event is shown with both a muon and recoil proton track.

$6 \times 10^{5}$ with typical pixel-pixel uniformity of $1: 2.5$ and cross talk of $4 \%$ for adjacent pixels.

\subsubsection{IRM boards}

The proposed FINeSSE experiment [14] specified a detector called SciBath [20] consisting of $\approx 19 \mathrm{k}$ WLS fibers immersed in liquid scintillator. In order to demonstrate the viability of this technique, an 800-fiber, $0.5 \mathrm{~m}^{3}$ prototype was built using Indiana University and NSF funding within the experimental nuclear physics group at the IU Cyclotron Facility (IUCF). This prototype included custom-built readout electronics for the 64-anode MAPMT. The high channel-count of this type of detector motivated a readout design using off-the-shelf components to keep the costs low. This was facilitated by the fact that data rates from a neutrino experiments are low and do not require a fast parallel readout bus.

This effort has resulted in the production of the Scibath Integrated Readout Module (IRM). The IRM consists of a $6 \mathrm{U}$ VME board with an "integrated" 64-channel multianode photomultiplier (MAPMT). A photo of a completed board is shown in Fig. 15 and the readout architecture is shown in Fig. 16.

The signal from each of the 64 MAPMT channels is routed to a "ringing integrator" analog 


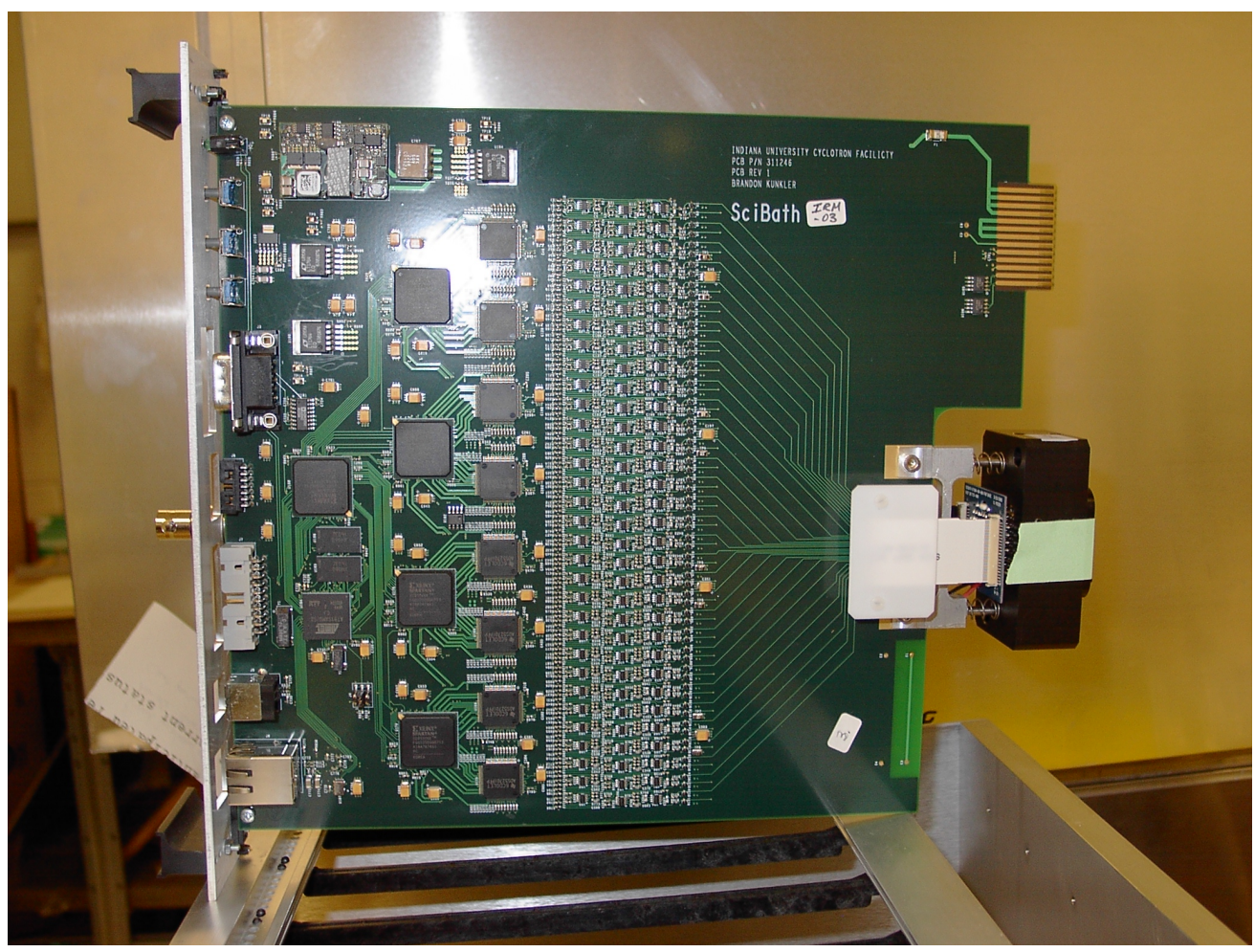

Figure 15: The SciBath Integrated Readout Module.

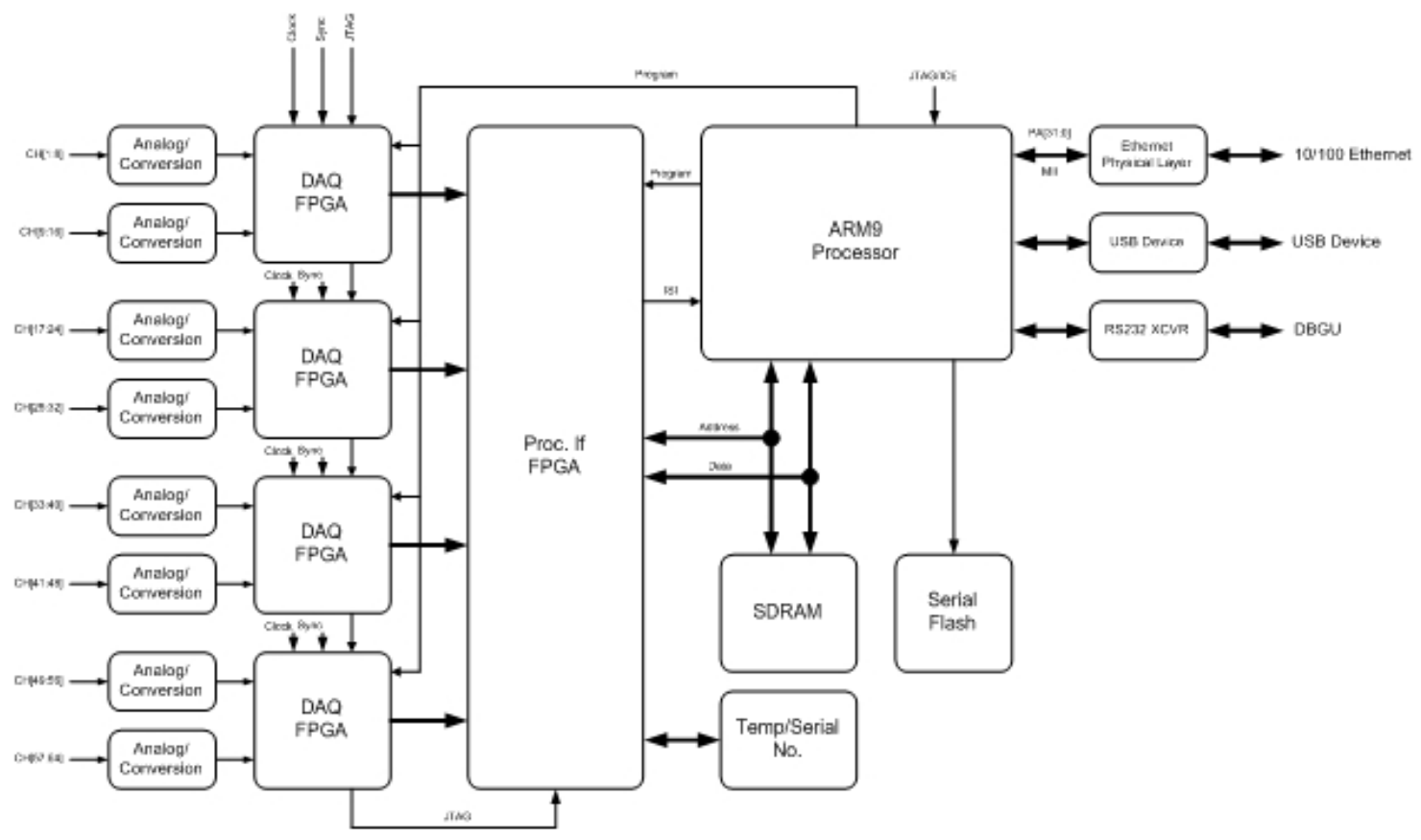

Figure 16: The IRM board architecture. 

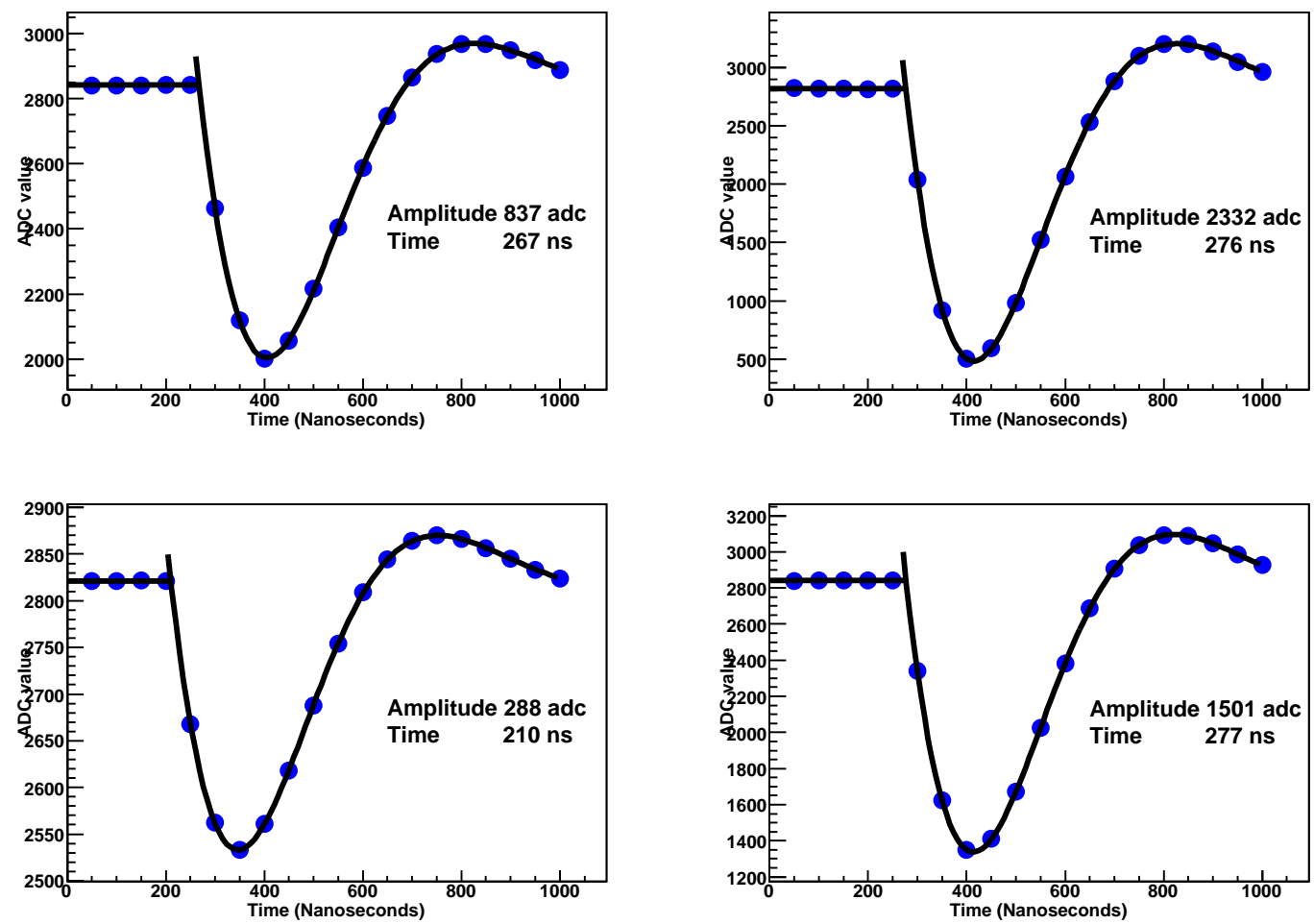

Figure 17: Four example MAPMT waveforms collected with the IRM modules with waveform fits superimposed. The extracted pulse amplitude and $T_{0}$ are indicated.

front-end circuit connected to a flash ADC running at $20 \mathrm{MHz}$. This front end circuit allows for both charge and time data to be obtained from a fit to the shaped PMT circuit. An example of several PMT waveforms collected with the existing IRM boards are shown in Fig. 17 . The amplitude of the fitted pulse provides the integrated PMT charge, proportional to the scintillator light output, and the phase of the fitted pulse provides the fine time or " $T_{0}$ " of the pulse. The full scale signal range is on order of 50 photoelectrons $(\mathrm{PE})$ with resolution $<0.1 \mathrm{PE}$. The timing resolution is $<10 \mathrm{~ns}$.

The ADC data is routed to a field programmable gate array ("DAQ" FPGA) where a decision is made in FPGA firmware whether to keep a particular ADC sample. There is one DAQ FPGA for 16 MAPMT channels, four per IRM. This decision is made in one of two modes. In "internal" trigger mode, the trigger for a particular channel is set if an ADC value is above a board-common threshold. In "external" mode, if a global trigger bit is set, then the trigger is set. In each of these cases, if the trigger is set, a programmable window of ADC data (currently 20 samples) is saved and routed to a FIFO for readout. We anticipate running in external mode to read out all data during a $10 \mu$ s beam spill.

A fifth "processor" FPGA (one per IRM) reads the buffered ADC data from the DAQ FPGAs into another FIFO buffer, one for each board. This buffer generates interrupts to an ARM9 microprocessor running Linux which reads and ships data upon request to an analysis ("host") computer via ethernet. This arrangement allows for fairly substantial on-board signal analysis and pattern recognition algorithms to be applied in quasi-realtime. The data is then sorted into events by the host computer which has access to all the data from the entire detector. 


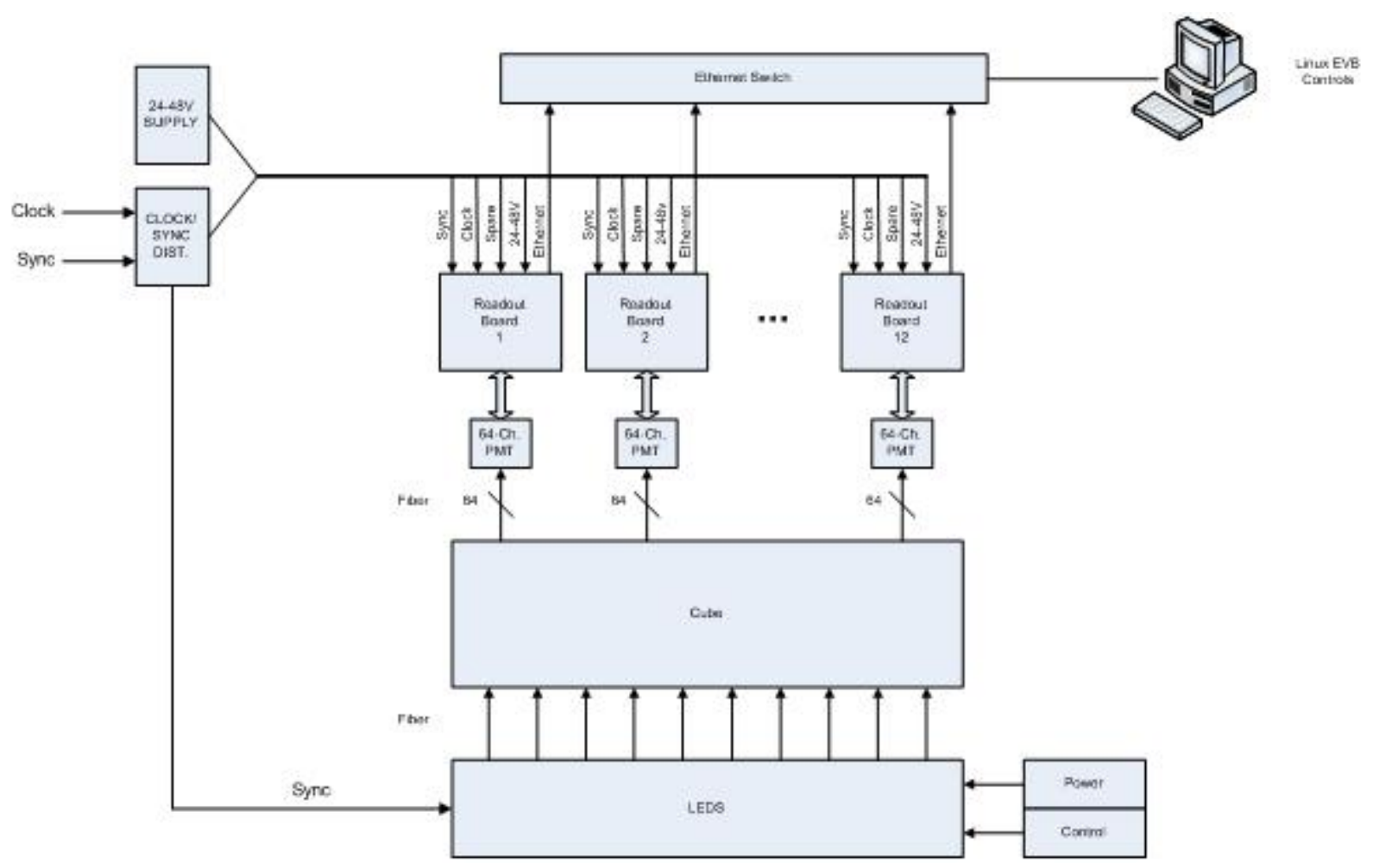

Figure 18: The IRM readout system.

The layout of the 12-board system attached to the prototype scibath detector is shown in Fig. 18. For a 232-board system, running with the SciNOvA detector, a similar scheme will be used. If all data from every MAPMT is readout during each $10 \mu$ s beam spill the resulting data rate will be 6 MBytes/spill. That is sufficiently low to be collected in one central computer. If desired that rate can be lowered by applying various criteria to the PMT waveforms at the FPGA, IRM microprocessor, or host computer level.

For the SciNOvA application, each IRM will be mounted in a standalone chassis and mounted on a support framework at the natural location of each of the 232 fiber cookies approximately $50 \mathrm{~cm}$ from the end of the scintillator bars. This scheme results in IRM boards spaced about $10 \mathrm{~cm}$ apart parallel to the beam and $20 \mathrm{~cm}$ transverse. Power and clock sync signals will be bussed to each board and driven with 4 custom-built clock/sync cards. These are fairly simple boards that provide the $20 \mathrm{MHz}$ signal that clocks the flash ADCs globally for the entire system and distributes the global trigger signal.

\subsection{Detector framework, assembly, and rigging}

The detector, including the PMTs and readout electronics housing, will be mounted in a FNALdesigned steel frame "cradle" and is enclosed in a dark box of approximate dimensions $4.1 \mathrm{~m}$ wide $\times 4.2 \mathrm{~m}$ tall $\times 2.3 \mathrm{~m}$ deep. The baseline plan is to build a new SciBar cradle based on the design for the original SciBar detector shown in Fig. 19

The SciBar detector for SciNOvA will be assembled as was the original SciBar detector (used for $\mathrm{K} 2 \mathrm{~K}$ and SciBooNE). The individual scintillator extrusions are glued into layers, with 116 strips oriented to measure $X$ and 116 to measure $Y$ in each layer. These layers are then loaded into the 


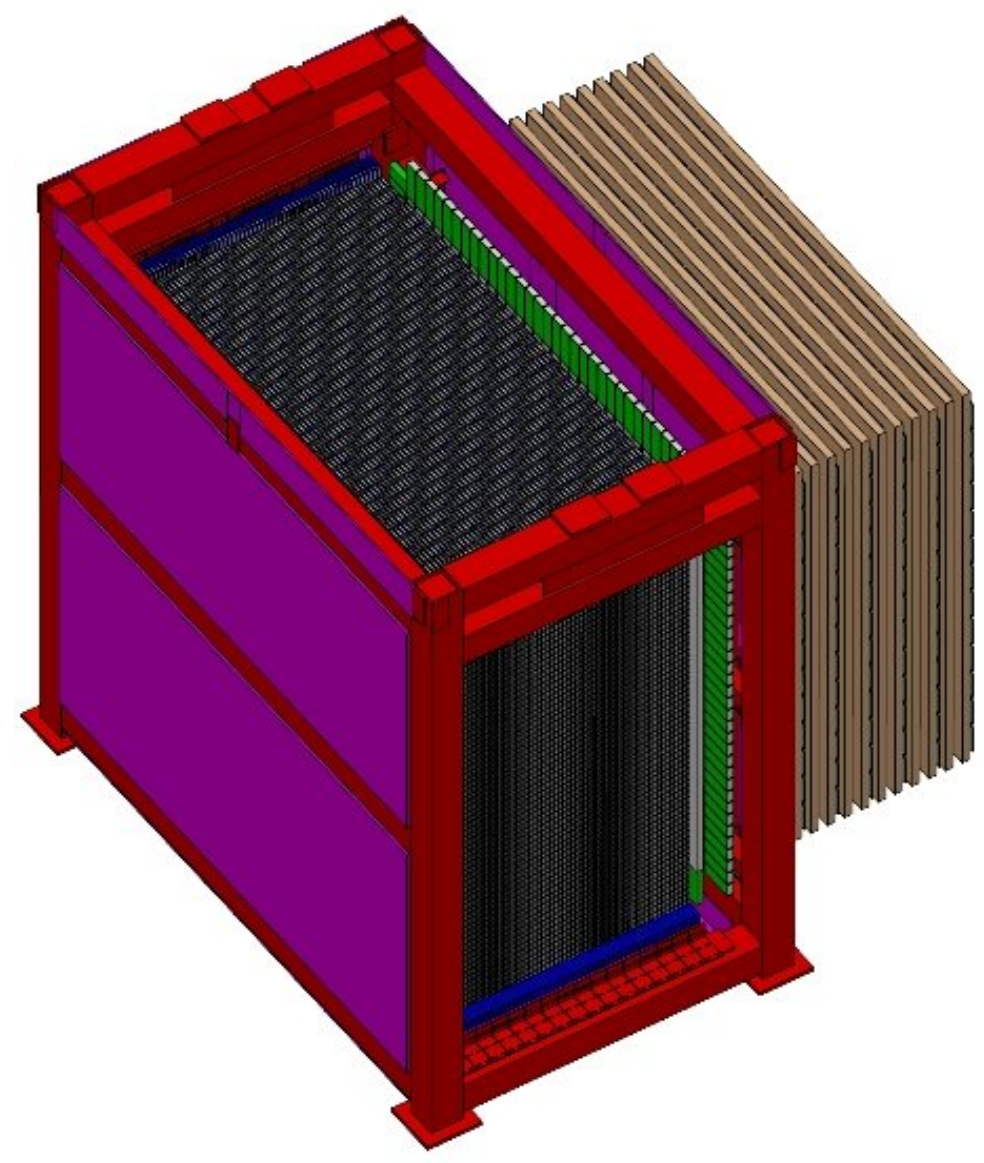

Figure 19: Isometric view of the SciBar detector within the SciBooNE detector framework. The SciBar frame is shown in red in this picture. The detector to the right in this figure outside of the frame is a muon range stack and will not be used in SciNOvA. The SciNOvA near detector will be used to measure forward muons that escape the SciBar detector. 


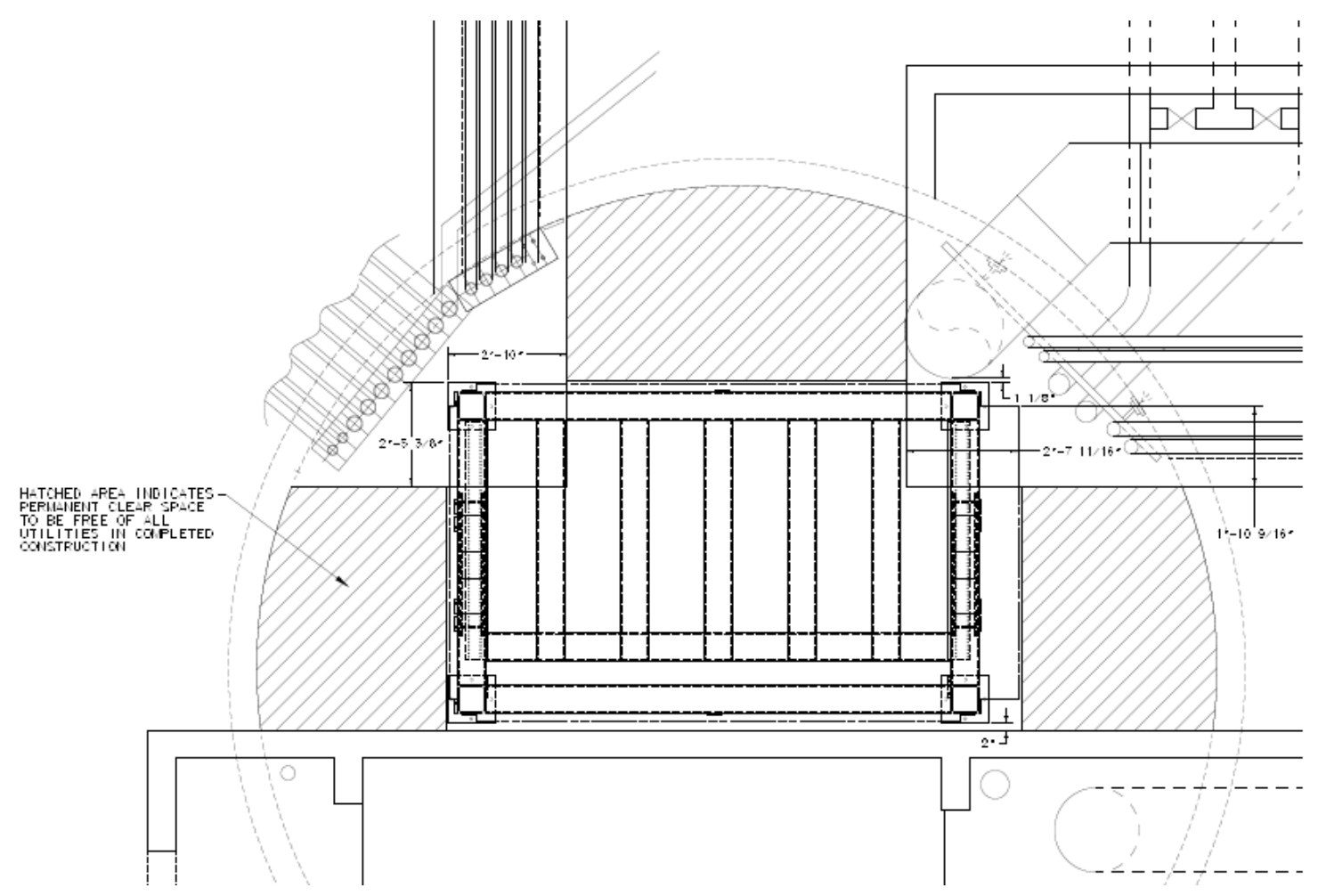

Figure 20: The SciBar cradle superimposed on the NUMI access shaft showing the clearance issues.

SciBar cradle to build up the entire SciBar detector consisting of 64 of these $X Y$ layers. This assembly process will require an open hall such as the CDF assembly area with crane coverage. We will load up the existing SciBar cradle with as many planes as possible while keeping the entire mass of the detector including the frame below the MINOS assembly building crane capacity of 15 tons. This partial assembly will be lowered down the NUMI access shaft and shuttled into a temporary assembly location. The remaining planes will be lowered in groups into the NUMI hall and installed into the SciBar cradle.

A study of possible assembly and installation issues was conducted by FNAL PPD engineering in June 2009 and concluded that there is adequate clearance to lower the SciBar cradle down the NUMI access shaft. However, the clearance is not generous (only a few inches at several points) as shown in Fig. 20. This issue will be addressed with modification of the SciBar cradle design. The SciBar cradle was designed to hold an electromagnetic calorimeter which will not be used for SciNOvA, and allowing for the cradle to be made shorter (top to bottom in Fig. 20) which would provide adequate clearance in the shaft.

The study also determined that there is adequate headroom at the top and bottom of the NUMI shaft to install the SciNOvA detector, and the plan for the NOvA near detector hall indicates that there is adequate space for the SciBar detector in front of the NOvA near detector (Fig. 12). However, interference with other support or scintillator containment structures will need to be identified and corrected to work with SciNOvA. There are sump pump covers in the MINOS hall that would need to be reinforced to allow the detector to be moved into position from the bottom of the access shaft. 


\section{Cost and schedule}

The SciNOvA project requires the construction and assembly of a new SciBar detector with new readout electronics (IRMs). These components and associated support structures must be installed in the NOvA near detector tunnel and are grouped with related tasks and summarized in the following list.

- SciBar detector:

- Scintillator extrusions. Constructed at FNAL using outside funds.

- Wavelength shifting (WLS) fibers. Purchased from Kurare (or similar vendor).

- WLS fiber bundles and cookies. Manufactured and assembled by collaborating institutions (not FNAL).

- SciBar framework. Holds scintillator extrusions, electronics. It includes dark box to surround all. This is assumed to be new item but using the SciBooNE design. The "strongback" cradle will be manufactured off-site with FNAL engineering support. Other components will be built by collaborating institutions.

- SciBar assembly and rigging. The majority of SciBar detector assembly will be performed by people supported by collaborating institutions. Support from FNAL for assembly space and rigging will be required.

- Integrated Readout Modules (IRMs)

- 64 anode PMTs with bases and mounting fixtures. PMTs purchased from Hamamatsu and tested by collaborating institutions. Bases and mounting fixtures assembled or subcontracted by collaborating institutions.

- IRM electronics boards. These are based on an existing design and will be anufactured off-site under supervision of collaborating institutions.

- Chassis for IRM and mounting fixtures. These will be purchased and assembled or fabricated by collaborating institutions.

- Power system for IRMs. This includes both low- and high-voltage systems to power IRMs and PMTs. This will be purchased/manufactured by collaborating institutions.

- Clock/trigger card system to drive IRMs. This will also use an existing and tested design. It will be assembled or subcontracted by collaborating institutions.

- DAQ computers and ethernet equipment. These items will be purchased by collaborating institutions.

- Detector installation. This includes engineering, rigging, fabrication work required to install the SciBar detector into the NOvA near detector area. This work is expected to be provided by FNAL.

In this assembly and installation plan, it is assumed that support is provided by FNAL for rigging during the SciNOvA assembly; and for rigging, engineering, fabrication during installation of the detector into the NOvA near detector hall. The plan at present is to seek funding for the remaining items from outside sources. 


\subsection{Cost estimate}

The estimated total costs for the project are summarized in Table 5. The estimated costs that FNAL would cover in this scenario are broken out in the rightmost column. The costs for scintillator extrusions come from an estimate provided by Anna Pla-Dalmau, which is shown in Fig. 21. Note that this estimate was for $12 \mathrm{k} 3 \mathrm{~m}$ scintillator bars and was scaled to estimate costs for a $15 \mathrm{k}$-bar SciBar detector for NOvA. FNAL indirect costs are included in this item.

This pricing assumes refabricating the scibar cradle and all lifting fixtures. If those make for the SciBooNE experiment can be recouped that would save approximately $\$ 100-150 \mathrm{k}$ depending on engineering cost to design modification. These costs were checked with those from the SciBooNE experiment. No contingencies or other indirect costs have been applied to these numbers.

\begin{tabular}{|c|c|c|c|}
\hline Item & costs (\$) & totals(\$) & $\begin{array}{l}\text { est FNAL } \\
\text { costs (\$) }\end{array}$ \\
\hline scibar & & 804818 & \\
\hline extrusions: $15 \mathrm{k} 3 \mathrm{~m}$ strips, $2.5 \mathrm{~cm} \times 1.3 \mathrm{~cm}$ & 410218 & & \\
\hline WLS fiber: 48km@\$2/m & 192000 & & \\
\hline fiber/PMT cookie assemblies & 25000 & & \\
\hline fabricate new scibar cradle & 120000 & & 120000 \\
\hline HVAC system & 8000 & & 8000 \\
\hline material and fab for assembly, lifting jigs & 24000 & & 24000 \\
\hline labor: assembly rigging & 25600 & & 25600 \\
\hline IRMs & & 1465770 & \\
\hline assembled boards: 250 & 1106028 & & \\
\hline clock board system & 3380 & & \\
\hline IRM power system & 26212 & & \\
\hline DAQ computer/enet hardware & 40000 & & \\
\hline elec design/testing/debug for IRMs & 87900 & & \\
\hline mechanical design for IRMs & 58600 & & \\
\hline final board assembly, repair & 37400 & & \\
\hline DAQ firmware, software & 106250 & & \\
\hline detector installation & & 141800 & \\
\hline engineering & 51200 & & 51200 \\
\hline rigging & 25600 & & 25600 \\
\hline material and fab for installation, lifting jig & 30000 & & 30000 \\
\hline misc underground infrastructure & 35000 & & 35000 \\
\hline project total & & 2412389 & 319400 \\
\hline
\end{tabular}

Table 5: Itemized costs for the SciNOvA project. Nominal FNAL costs are listed in the rightmost column.

A total of 250 IRM board (232 plus spares) are required. A breakdown of the IRM board costs are provided in Table 6. Note that the MAPMTs are included here as are PMT base boards the integrated HV supply and all mounting fixtures. The cost/board tallies to approximately $\$ 4.4 \mathrm{k}$ with cost/channel at $\$ 69$. Again, these costs include PMTs. 


\section{COST ESTIMATE FOR SCINOVA}

Requested by Mark Messier at Indiana University

Prepared by Anna Pla-Dalmau

Date: May 13, 2010

IMPORTANT: PROJECT WILL BE BILLED AT ACTUAL COSTS. THIS IS AN ESTIMATE.

Scintillator bars with titanium dioxide coating with one hole for a WLS fiber: $2.5 \mathrm{~cm} \times 1.3 \mathrm{~cm}$ at $300 \mathrm{~cm}$ Total amount of scintillator: $36,000 \mathrm{~m}(12,000$ strips $)$

\begin{tabular}{|c|c|c|c|c|c|}
\hline & $\begin{array}{l}\text { Estimated } \\
\text { Materials } \\
\text { and } \\
\text { Services } \\
\text { Cost (\$) }\end{array}$ & $\begin{array}{c}\text { Estimate } \\
\text { d Time } \\
\text { (hours) }\end{array}$ & $\begin{array}{c}\text { Rate } \\
\text { (hours) }\end{array}$ & $\begin{array}{c}\text { Estimated } \\
\text { Labor Cost } \\
\text { (\$) }\end{array}$ & \begin{tabular}{|c|} 
Total \\
Estimated \\
Cost \\
Materials \\
and Labor \\
(\$)
\end{tabular} \\
\hline \multicolumn{6}{|l|}{$R \& D$ Material } \\
\hline Die & $\$ 10,000.00$ & & & & \\
\hline Polystyrene pellets (1,480 Kg @ \$2.65 each) & $\$ 3,922.00$ & & & & \\
\hline Dopants (34 bottles @ \$190 each) & $\$ 6,460.00$ & & & & \\
\hline Titanium dioxide pellets (40 Kg @ \$7.85 each) & $\$ 314.00$ & & & & \\
\hline Nitrogen gas (10 LN ${ }_{2}$ dewars @ \$126 each) & $\$ 1,260.00$ & & & & \\
\hline Consumables (jars, labels, QC tools, $\ldots$ ) & $\$ 1,000.00$ & & & & \\
\hline \multicolumn{6}{|l|}{ R\&D Labor } \\
\hline Extrusion preparation and operation & & 120 & $\$ 60.00$ & $\$ 7,200.00$ & \\
\hline Extrusion assistance & $\$ 3,400.00$ & 100 & $\$ 34.00$ & & \\
\hline Extrusion assistance and QC & & 60 & $\$ 35.00$ & $\$ 2,100.00$ & \\
\hline Set-up and tear-down (half-day each, 2 people) & & 20 & $\$ 60.00$ & $\$ 1,200.00$ & \\
\hline \multicolumn{6}{|l|}{ Production Material } \\
\hline Polystyrene pellets $(14,800 \mathrm{Kg} @ \$ 2.65$ each) & $\$ 39,220.00$ & & & & \\
\hline Dopants (340 bottles @ \$190 each) & $\$ 64,600.00$ & & & & \\
\hline Titanium dioxide pellets (400 Kg @ \$7.85 each) & $\$ 3,140.00$ & & & & \\
\hline Nitrogen gas (50 LN 2 dewars @ \$126 each) & $\$ 6,300.00$ & & & & \\
\hline Consumables (jars, labels, QC tools,...) & $\$ 1,500.00$ & & & & \\
\hline \multicolumn{6}{|l|}{ Production Labor } \\
\hline Extrusion preparation and operation & & 800 & $\$ 60.00$ & $\$ 48,000.00$ & \\
\hline Extrusion assistance & $\$ 14,960.00$ & 440 & $\$ 34.00$ & & \\
\hline Extrusion assistance and QC & & 400 & $\$ 35.00$ & $\$ 14,000.00$ & \\
\hline Project coordination & & 80 & $\$ 65.00$ & $\$ 5,200.00$ & \\
\hline Set-up and tear-down (half-day each, 2 people) & & 20 & $\$ 60.00$ & $\$ 1,200.00$ & \\
\hline \multicolumn{6}{|l|}{ Crating and Shipping } \\
\hline Crate - 12 wooden crates & $\$ 3,600.00$ & & & $\$ 3,600.00$ & \\
\hline Shipping* & $\$ 6,000.00$ & & & & \\
\hline Extrusion Equipment Maintenance & $\$ 3,000.00$ & & & & \\
\hline Estimated Direct Cost & $\$ 168,676.00$ & & & $\$ 82,500.00$ & $\$ 251,176.00$ \\
\hline FNAL Indirect Charges (14.4\% M\&S) & $\$ 24,289.34$ & & & & $\$ 24,289.34$ \\
\hline TOTAL Estimated Cost & & & & $\$ 5<, 109 .<5$ & $\$ 328,174.59$ \\
\hline
\end{tabular}

*This is an estimate.

Figure 21: Scintillator extrusion cost estimate. 


\begin{tabular}{|lrrr|}
\hline & \multicolumn{3}{c|}{ costs } \\
IRM component & qty & each & total \\
\hline assembled PCB with components & 250 & $\$ 2,515.00$ & $\$ 628,750$ \\
integrated HV supply & 250 & $\$ 32.63$ & $\$ 8,158$ \\
MAPMTs & 250 & $\$ 1,600.00$ & $\$ 400,000$ \\
PMT base PCB assembly & 250 & $\$ 95.00$ & $\$ 23,750$ \\
PMT mounting parts, ribbon cable & 250 & $\$ 142.84$ & $\$ 35,710$ \\
chasis mounting parts, connectors & 250 & $\$ 15.71$ & $\$ 3,928$ \\
Fans & 250 & $\$ 22.93$ & $\$ 5,733$ \\
\hline total IRM costs & & & $\mathbf{\$ 1 , 1 0 6 , 0 2 8}$ \\
cost/board & & & $\$ 4,424$ \\
cost/channel & & & $\$ 69.13$ \\
\hline
\end{tabular}

Table 6: Breakout of components and costs for the IRM boards based on manufacture of 250 boards.

\subsection{Schedule}

The schedule for building and installing the SciNOvA detector is shown in Table 7 . The start of the project is estimated to be 10/1/11 with start of funding. This is an estimate based on a possible NSF MRI funding scenario. It is possible that, even if funding starts on this date, parts of the project may be started earlier if resources can be available earlier. The project ready date is that of completion of detector installation in the NOvA near hall and is estimated to be 23 months after the project start. 


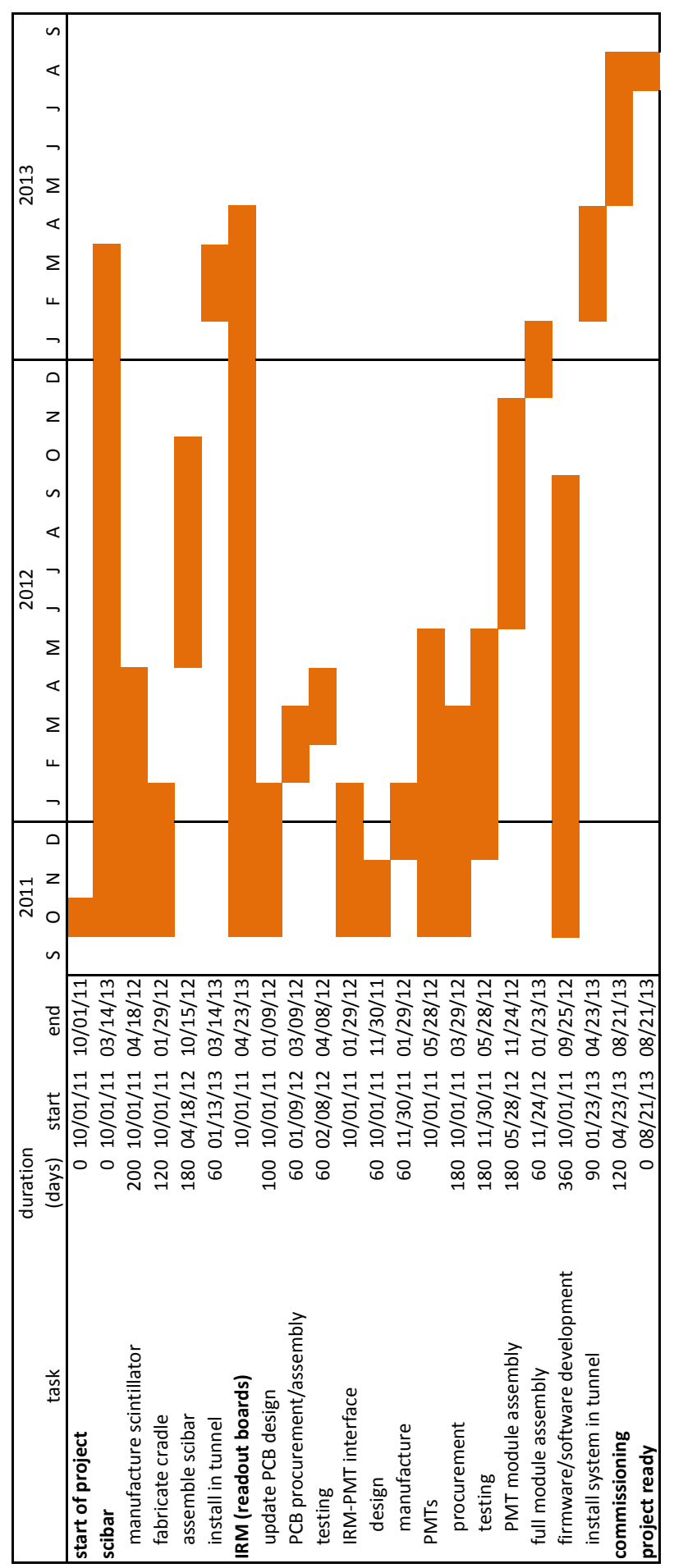

Table 7: Construction and installation schedule for SciNOvA. 


\section{References}

[1] C. Andreopoulos et al., arXiv:0905.2517 [hep-ph].

[2] V. Lyubushkin et al. [NOMAD Collaboration], Eur. Phys. J. C 63, 355 (2009) [arXiv:0812.4543 [hep-ex]].

[3] V. Bernard, L. Elouadrhiri and U. G. Meissner, J. Phys. G 28, R1 (2002) [arXiv:hep$\mathrm{ph} / 0107088]$.

[4] A. A. Aguilar-Arevalo et al. [MiniBooNE Collaboration], Phys. Rev. Lett. 100, 032301 (2008); Phys. Rev. D 81, 092005 (2010).

[5] K. L. Miller et al., Phys. Rev. D 26, 537 (1982).

[6] L. Alvarez-Ruso, O. Buss, T. Leitner and U. Mosel, arXiv:0909.5123 [nucl-th].

[7] J. L. Alcaraz-Aunion and J. Walding, arXiv:0909.5647 [hep-ex].

[8] D. Casper, Nucl. Phys. Proc. Suppl. 112, 161 (2002).

[9] M. Martini et al., Phys. Rev. C81, 045502 (2010); Phys. Rev. C80, 065501 (2009).

[10] J. E. Amaro et al., arXiv:1010.1708v1 [nucl-th] (2010).

[11] R. Subedi et al., Science 320, 1476 (2008) [arXiv:0908.1514 [nucl-ex]].

[12] A. A. Aguilar-Arevalo et al. [MiniBooNE Collaboration], Phys. Lett. B 664, 41 (2008) [arXiv:0803.3423 [hep-ex]].

[13] K. Hiraide et al. [SciBooNE Collaboration], Phys. Rev. D 78, 112004 (2008) [arXiv:0811.0369 [hep-ex]].

[14] L. Bugel et al. [FINeSSE Collaboration], arXiv:hep-ex/0402007.

[15] US Particle Physics: Scientific Opportunities, A Strategic Plan for the Next Ten Years. Report of the Particle Physics Project Prioritization Panel, June 2008. http://www.science.doe.gov/hep/panels/reports/hepap_reports.shtml

[16] A. A. Aguilar-Arevalo et al. [LSND Collaboration], Phys. Rev. D64, 112007 (2001).

[17] A. A. Aguilar-Arevalo et al. [MiniBooNE Collaboration], Phys. Rev. Lett. 98, 231801 (2007); Phys. Rev. Lett. 102, 101802 (2009); Phys. Rev. Lett. 103, 111801 (2009); arXiv:1007.1150 [hep-ex].

[18] K. Nitta et al., Nucl. Instrum. Meth. A 535, 147 (2004) [arXiv:hep-ex/0406023].

[19] A. A. Aguilar-Arevalo et al. [SciBooNE Collaboration], arXiv:hep-ex/0601022.

[20] R. Tayloe et al., Nucl. Instrum. Meth. A 562, 198 (2006).

[21] FERMILAB-PUB-05-344, http://nicadd.niu.edu/research/extruder/extruder_files/ doc/PUBLICATIONS/fermilab-pub-05-344.pdf

[22] http://minerva.fnal.gov/ 